\title{
MIXED GROUPS
}

\author{
PAUL HILL AND CHARLES MEGIBBEN
}

\begin{abstract}
As the culmination of a series of several papers, we establish here a combinatorial characterization of Warfield groups (that is, direct summands of simply presented abelian groups) in terms of knice subgroups-a refinement of the concept of nice subgroup appropriate to the study of groups containing elements of infinite order. Central to this theory is the class of $k$-groups, those in which 0 is a knice subgroup, and the proof that this class is closed under the formation of knice isotype subgroups. In particular, a direct summand of a $k$-group is a $k$-group. As an application of our Axiom 3 characterization of Warfield groups, we prove that $k$-groups of cardinality $\aleph_{1}$ have sequentially pure projective dimension $\leq 1$; or equivalently, if $H$ is a knice isotype subgroup of the Warfield group $G$ with $|G / H|=\aleph_{1}$, then $H$ is itself a Warfield group.
\end{abstract}

\section{INTRODUCTION}

In this paper, we provide the long-promised proof of the Axiom 3 characterization of global Warfield groups first sketched in our Colorado Springs lectures [HM5]. Much of the technical groundwork for this development appeared in our Oberwolfach lectures [HM4]. In broad outline, the ideas underlying this characterization are by now fairly well known. This is particularly true in view of our previous publication [HM2] of the $p$-local version of the theory. It is convenient to assume at the outset that all groups are abelian. Recall that a simply presented group is one that can be given in terms of generators and relations in such a manner that the relations involve at most two generators, and that a Warfield group is defined to be a direct summand of a simply presented (mixed) group. Most of the theory of Warfield groups, however, was developed by Hunter, Richman and Walker [HRW1, HRW2, HR] using an alternative definition; to wit, a mixed group $G$ is called a Warfield group provided it contains a nice decomposition basis $X$ such that $G /\langle X\rangle$ is a totally projective group. This latter approach has two notable advantages. Firstly, it yields rather quickly via the Hill-Walker theorem the uniqueness theorem for Warfield groups (although some set-theoretical gymnastics with decomposition bases are required); and secondly, it leads rather naturally to an existence the-

Received by the editors August 15, 1990 .

1980 Mathematics Subject Classification (1985 Revision). Primary 20K21, 20 K27.

Key words and phrases. Mixed groups, Axiom 3 characterization, Warfield groups, simply presented groups, knice subgroups, $k$-groups, primitive element, decomposition basis, sequential purity.

The authors were supported in part, respectively, by NSF grants DMS-8800862 and DMS8900235. 
ory for these groups. However, part of the price paid for this approach is that closure under direct summands is no longer automatic and, in fact, becomes a major problem. Warfield [W] had earlier suggested, in the spirit of Hill's characterization of totally projective $p$-groups [H1], the possibility of an Axiom 3 characterization for direct summands of simply presented groups, but he obtained a satisfactory result only in the rank 1 case. One should understand that there are several reasons for desiring such a characterization. For example, it would provide a basis for a more natural proof, via finite extensions, of the uniqueness theorem. More importantly, the combinatorial techniques that arise from Axiom 3 characterizations could be applied to the study of subgroups of Warfield groups, and equivalence theorems such as that of [HM1] would likely give further insight into the structure of isotype subgroups of simply presented groups. In fact, our Axiom 3 approach to $p$-local Warfield groups in [HM2 and HM7] has largely fulfilled these expectations. However, the desirability of such an approach and the availability of the general outline notwithstanding, the transition from $p$-local to global Warfield groups is by no means routine (for example, constrast the treatment of the summand problem in [HRW1 and AHR]). Accordingly we found it helpful to deal first with torsion-free groups [HM3]. In spite of the fact that in this context simply presented groups are completely decomposable and hence seemingly of transparent structure, our concept of primitive element and of $*$-valuated coproduct (first introduced for $p$-local groups in [HM2]) have shed light on questions dating back to Baer's fundamental paper [B]. For example, in a torsion-free separable group $G$, the subgroup $\left\langle x_{1}\right\rangle \oplus \cdots \oplus\left\langle x_{n}\right\rangle$ is a $*$-valuated coproduct with the $x_{i}$ 's primitive in $G$ if and only if $\left\langle x_{1}\right\rangle_{*} \oplus \cdots \oplus\left\langle x_{n}\right\rangle_{*}$ is a direct summand of $G$. Furthermore, even though an Axiom 3 characterization of completely decomposable groups might seem on the surface a frivolous exercise, we point to [DR and HM6] as illustrations of interesting consequences of this characterization. A general equivalence theorem for pure subgroups of completely decomposable groups has yet to be established, but a special case is settled in [HM9] and these ideas have led to a solution in [HM8] of an old problem concerning the equivalence of different free resolutions of a given group (Problem 36 in [F1]).

The central idea on which this paper rests is the formulation of a suitable notion of "knice subgroup" (see the definition at the beginning of $\S 3$ ). The global mixed groups satisfying Axiom 3 with respect to these subgroups are precisely the global Warfield groups. In contrast to the fact that 0 is always a nice subgroup, we remark that 0 being a knice subgroup of $G$ has nontrivial structural consequences. Indeed we reserve the term $k$-group for groups in which 0 is a knice subgroup. The underlying intuition is that a $k$-group has local decomposition bases in the sense that each finitely generated subgroup is a finite extension of a special sort of free-valuated group. The exact definition of kniceness will depend on the auxiliary notions of primitive element and *-valuated coprod$u c t$. As indicated earlier, the appropriate definitions of these concepts for the global case appeared in [HM4]; the remainder of this introductory section is largely devoted to a recapitulation of the salient facts from the paper just cited. However, in this introduction, we also include some new results. For example, Proposition 1.7 contains an alternative characterization of knice subgroups that has not heretofore appeared, and Proposition 1.9 is a specialization of this result to subgroups that are both knice and isotype. 
We first establish the notation that will be in effect throughout this paper, deferring to [HM4] for a more detailed discussion. We let $\mathscr{O}_{\infty}$ denote the class of ordinals with the symbol $\infty$ adjoined as a maximum element, and we adopt the convention that $\infty<\infty$. By an abstract height, we mean a sequence $s=\left(s_{p}\right)_{p \in \mathbb{P}}$ where $\mathbb{P}$ denotes the set of rational primes and each $s_{p}$ is an element of $\mathscr{O}_{\infty}$. With each element $x$ of a mixed group $G$, we associate its height $|x|=$ $\left(|x|_{p}\right)_{p \in \mathbb{P}}$ where $|x|_{p}$ is the $p$-height of $x$ as computed in $G$. We also associate with each $x \in G$ its height matrix $\|x\|$, the doubly infinite $\mathbb{P} \times \omega$ matrix having $\left|p^{i} x\right|_{p}$ as its $(p, i)$ entry. Occasionally we shall affix a subscript to $\|x\|$ in order to emphasize the particular group in which heights are computed. More generally, we consider abstract height matrices $M=\left[m_{p, i}\right]_{(p, i) \in \mathbb{P} \times \omega}$ with entries from $\mathscr{O}_{\infty}$ and with the condition $m_{p, i}<m_{p, i+1}$ satisfied for all $p$ and all $i$. We write $M_{p}$ to denote the $p$-row of the height matrix $M$, and thus $\|x\|_{p}$ is the sequence $\left(\left|p^{i} x\right|_{p}\right)_{i<\omega}$. Allowing positive integers to operate on abstract height matrices in the obvious manner, we have $n\|x\|=\|n x\|$ for all positive integers $n$ and all $x \in G$. The order structure of $\mathscr{O}_{\infty}$, of course, induces in a point-wise fashion lattice relations $\leq$ and $\wedge$ on the classes of heights and height matrices. Two height matrices $M$ and $M^{\prime}$ are said to be quasi-equivalent, and we write $M \sim M^{\prime}$, provided there exist positive integers $m$ and $n$ such that $m M^{\prime} \geq M$ and $n M \geq M^{\prime}$. Notice that $M^{\prime} \sim M^{\prime}$ implies that $M_{p}=M_{p}^{\prime}$ for all but finitely many primes $p$. With each height matrix $M$ and each mixed group $G$, we associate the fully invariant subgroups $G(M)=\{x \in G:\|x\| \geq M\}$ and $G\left(M^{*}\right)=\langle x \in G(M):\|x\| \nsim M\rangle$. (In order to accommodate nonreduced groups, we make a slight exception to the last definition, namely, if $M$ is quasiequivalent to the height matrix with all entries $\infty$, then we take $G\left(M^{*}\right)$ to be the maximal torsion subgroup of $G(M)$.) For each prime $p$ and each sequence $\alpha=\left(\alpha_{i}\right)_{i<\omega}$ in $\mathscr{O}_{\infty}$ with $\alpha_{i}<\alpha_{i+1}$ for all $i$, we define $G\left(\alpha^{*}, p\right)$ to be that subgroup generated by those elements $x \in G$ such that $\left|p^{i} x\right|_{p} \geq \alpha_{i}$ for all $i$ and $\left|p^{i} x\right|_{p} \neq \alpha_{i}$ for infinitely many values of $i$. Finally, for each height matrix $M$ and each prime $p$, we let $G\left(M^{*}, p\right)=G(M) \cap\left[G\left(M^{*}\right)+G\left(M_{p}^{*}, p\right)\right]$.

Definition 1.1. An element $x$ of the mixed group $G$ is said to be a primitive element if, for each positive integer $n$, each height matrix $M$ and each prime $p, n x \in G\left(M^{*}, p\right)$ implies either $\|x\| \nsim M$ or $\left|p^{i} n x\right|_{p} \neq m_{p, i}$ for infinitely many values of $i$.

Observe that primitive elements have infinite order. Also, for any positive integer $n$, the element $x$ is primitive if and only if $n x$ is primitive (Proposition 2.2 of [HM4]).

If $\left(A_{i}\right)_{i \in I}$ is a family of independent subgroups of the group $G$, then the direct sum $A=\bigoplus_{i \in I} A_{i}$ is said to be a valuated coproduct in $G$ provided that $|a|=\bigwedge_{i \in I}\left|a_{i}\right|$ whenever $a=\sum_{i \in I} a_{i}$ with $a_{i} \in A_{i}$. An equivalent formulation of this concept is that $A \cap G(M)=\bigoplus_{i \in I}\left(A_{i} \cap G(M)\right)$ for all height matrices $M$.

Definition 1.2. Let $\left(A_{i}\right)_{i \in I}$ be a family of independent subgroups of the mixed group $G$. We say that $A=\bigoplus_{i \in I} A_{i}$ is a *-valuated coproduct in $G$ provided that $A \cap F=\bigoplus_{i \in I}\left(A_{i} \cap F\right)$ for all fully invariant $F$ of the form $G(M)$, $G\left(M^{*}\right), G\left(\alpha^{*}, p\right)$, or $G\left(M^{*}, p\right)$.

If $A=\bigoplus_{i \in I} A_{i}$ is a valuated coproduct in $G$ and if $\left(B_{i}\right)_{i \in I}$ is a family of 
subgroups such that $A_{i} / B_{i}$ is torsion for all $i$ and $B=\bigoplus_{i \in I} B_{i}$ is a $*$-valuated coproduct in $G$, then it can be shown that $A=\bigoplus_{i \in I} A_{i}$ itself is a *-valuated coproduct. The proof depends on the fundamental fact that if $g \in G(M)$ and if, for some positive integer $n, n g \in G\left(n M^{*}, p\right)$, then $g \in G\left(M^{*}, p\right)$. In order to place these new concepts in a context that may be more familiar to the reader, we recall that a subset $X=\left(x_{i}\right)_{i \in I}$ of elements of infinite order in the mixed group $G$ is said to be a decomposition basis if $G /\langle X\rangle$ is a torsion group and $\langle X\rangle=\bigoplus_{i \in I}\left\langle x_{i}\right\rangle$ is a valuated coproduct in $G$. It is not difficult to show that if $X=\left(x_{i}\right)_{i \in I}$ is a decomposition basis for $G$, then each $x_{i}$ is a primitive element of $G$ and that $\langle X\rangle=\bigoplus_{i \in I}\left\langle x_{i}\right\rangle$ is indeed a $*$-valuated coproduct. In the next proposition, we collect from [HM4] a list of those properties of primitive elements and *-valuated coproducts that we shall most frequently use in the present paper.

Proposition 1.3. (1) If $x$ is a primitive element of the mixed group $G$ with $\|x\|=M$ and if $y$ is contained in the coset $x+G\left(M^{*}\right)$, then $y$ is also a primitive element and there is a positive integer $n$ such that $\|n y\|=\|n x\|$.

(2) Suppose $A=B \oplus\langle x\rangle$ is a *-valuated coproduct in the mixed group $G$ with $x$ a primitive element. If $y=z+x$ where $z \in B$ and $\|y\|=\|x\|$, then $y$ is a primitive element and $A=B \oplus\langle y\rangle$ is a *-valuated coproduct.

(3) If $x=x_{1}+x_{2}+\cdots+x_{n}$ where $C=\left\langle x_{1}\right\rangle \oplus\left\langle x_{2}\right\rangle \oplus \cdots \oplus\left\langle x_{n}\right\rangle$ is a *-valuated coproduct in the mixed group $G$ and the $x_{i}$ 's are mutually quasi-equivalent primitive elements, then $x$ is a primitive element of $G$ if and only if, for each prime $p$, there is some $s<\omega$ and some $i \leq n$ such that $\left\|p^{s} x\right\|_{p}=\left\|p^{s} x_{i}\right\|_{p}$.

(4) Suppose that both $A \oplus\left\langle y_{1}\right\rangle$ and $B=A \oplus\left\langle x_{1}\right\rangle \oplus \cdots \oplus\left\langle x_{m}\right\rangle$ are $*$-valuated coproducts in the mixed group $G$ where $y_{1}$ and all the $x_{i}$ 's are primitive elements. If $y_{1} \in B$, then there exist primitive elements $y_{2}, \ldots, y_{m}$ such that $\boldsymbol{B}^{\prime}=A \oplus\left\langle y_{1}\right\rangle \oplus\left\langle y_{2}\right\rangle \oplus \cdots \oplus\left\langle y_{m}\right\rangle$ is a *-valuated coproduct with $\boldsymbol{B} / \boldsymbol{B}^{\prime}$ finite.

Parts (1), (2) and (4) of Proposition 1.3 are, respectively, Proposition 2.4, Proposition 2.6, and Theorem 2.10 of [HM4]; while (3) is established in the discussion immediately following the proof of Lemma 2.8 of that paper.

Corollary 1.4. Suppose that both $\left\langle y_{1}\right\rangle \oplus \cdots \oplus\left\langle y_{m}\right\rangle$ and $C=\left\langle x_{1}\right\rangle \oplus \cdots \oplus\left\langle x_{n}\right\rangle$ are *-valuated coproducts in the mixed group $G$ where the $x_{i}$ 's and $y_{j}$ 's are all primitive elements. If all the $y_{j}$ 's are contained in $C$, then there exist primitive elements $z_{1}, \ldots, z_{k}$ with $k=n-m$ such that $C^{\prime}=\left\langle y_{1}\right\rangle \oplus \cdots \oplus\left\langle y_{m}\right\rangle \oplus\left\langle z_{1}\right\rangle \oplus$ $\cdots \oplus\left\langle z_{k}\right\rangle$ is a *-valuated coproduct with $C / C^{\prime}$ finite.

Proof. By induction on $m$. The case $m=1$ follows immediately from Proposition 1.3(4) with $A=0$. Assume that the result holds for $m-1$. Then applying Proposition 1.3(4) with $A=\left\langle y_{1}\right\rangle \oplus \cdots \oplus\left\langle y_{m-1}\right\rangle$, we obtain a $*$-valuated coproduct $C^{\prime}=\left\langle y_{1}\right\rangle \oplus \cdots \oplus\left\langle y_{m-1}\right\rangle \oplus\left\langle y_{m}^{\prime}\right\rangle \oplus\left\langle z_{1}\right\rangle \oplus \cdots \oplus\left\langle z_{k}\right\rangle$ where $y_{m}^{\prime}$ is a nonzero multiple of $y_{m}, C / C^{\prime}$ is finite and the elements $z_{1}, \ldots, z_{k}$ are primitive. The Seesaw Lemma, Proposition 2.7 in [HM4], allows us to replace $y_{m}^{\prime}$ by $y_{m}$ at the expense of replacing $z_{1}, \ldots, z_{k}$ by nonzero multiples of themselves.

The paper [HM4] also contains a new definition of global niceness, namely, $H$ is a nice subgroup of the group $G$ if, for all primes $p$ and all ordinals $\alpha$, the cokernel of the canonical map $\left(p^{\alpha} G+H\right) / H \rightarrow p^{\alpha}(G / H)$ contains no element of order $p$. If $G$ is a torsion group, this condition reduces to the familiar equation $p^{\alpha}(G / H)=\left(p^{\alpha} G+H\right) / H$ for all $p$ and $\alpha$. But for mixed groups, 
a larger class of nice subgroups is obtained than under the older definition via localization [HR]. In particular, under this new dispensation cyclic subgroups are nice and, more generally, if $A=\left\langle x_{1}\right\rangle \oplus \cdots \oplus\left\langle x_{n}\right\rangle$ is a valuated coproduct in $G$, then $A$ is a nice subgroup of $G$ (see Theorem 1.8 of [HM4]). For a subgroup $H$ with $G / H$ torsion, $H$ is nice under one definition if and only if it is nice under the other. In the present paper, we shall use exclusively the definition of nice subgroup given above.

Definition 1.5. A subgroup $A$ of the mixed group $G$ is said to be a knice subgroup provided the following two conditions are satisfied:

(1) $A$ is a nice subgroup of $G$.

(2) If $S$ is a finite subset of $G$, then there is a finite collection of primitive elements $x_{1}, \ldots, x_{m}$ in $G$ such that $A^{\prime}=A \oplus\left\langle x_{1}\right\rangle \oplus \cdots \oplus\left\langle x_{m}\right\rangle$ is a *-valuated coproduct in $G$ with $\left\langle S, A^{\prime}\right\rangle / A^{\prime}$ finite.

The most important facts concerning knice subgroups are summarized in our next proposition. As indicated below, all these results are proved in detail in [HM4].

Proposition 1.6. (1) If $A$ is a knice subgroup of the mixed group $G$ and if $A^{\prime}$ is a subgroup of $G$ with $A^{\prime} / A$ finite, then $A^{\prime}$ is a knice subgroup of $G$.

(2) If $A^{\prime}=A \oplus\left\langle x_{1}\right\rangle \oplus \cdots \oplus\left\langle x_{m}\right\rangle$ is a *-valuated coproduct in the mixed group $G$ where $A$ is a knice subgroup of $G$ and the $x_{i}$ 's are primitive elements of $G$, then $A^{\prime}$ is a knice subgroup of $G$.

(3) If $A$ is a knice subgroup of the mixed group $G$ and if $S$ is an arbitrary finite subset of $G$, then there is a knice subgroup $A^{\prime}$ of $G$ such that $A^{\prime} \supseteq\langle S, A\rangle$ and $A^{\prime} / A$ is finitely generated.

(4) If $A$ is a knice subgroup of the mixed group $G$ and if $B / A$ is a knice subgroup of the quotient group $G / A$, then $B$ is a knice subgroup of $G$.

Parts (1), (2), and (4) of Proposition 1.6 are, respectively, Theorem 3.2, 3.3, and 3.7 of [HM4]; and obviously (3) is a consequence of (1) and (2), together with Definition 1.5.

Recollect that we call $G$ a $k$-group if 0 is a knice subgroup. If $A$ is a knice subgroup of $G$, then it turns out not surprisingly that $G / A$ is a $k$-group. We shall, in fact, frequently need a characterization of a knice subgroup $A$ of $G$ that places emphasis on properties of the quotient group $G / A$.

Proposition 1.7. Let $A$ be a subgroup of the mixed group $G$. Then $A$ is a knice subgroup of $G$ if and only if the following three conditions are satisfied:

(1) The quotient group $G / A$ is a k-group.

(2) $A$ is a nice subgroup of $G$.

(3) To each $g \in G$ there corresponds a positive integer $n$ such that the coset $n g+A$ contains an element $x$ such that $\|x\|_{G}=\|n g+A\|_{G / A}$.

Proof. First suppose $A$ is a knice subgroup of the mixed group $G$. Then (2) holds by definition and (3) follows by Lemma 3.6 of [HM4]. To prove (1) it clearly suffices to establish the following: If $A \oplus\left\langle x_{1}\right\rangle \oplus \cdots \oplus\left\langle x_{m}\right\rangle$ is a $*$-valuated coproduct with the $x_{i}$ 's primitive elements in $G$, then $\left\langle x_{1}+A\right\rangle \oplus \cdots \oplus\left\langle x_{m}+A\right\rangle$ is a *-valuated coproduct with the $x_{i}+A$ 's primitive elements in $G / A$. We begin by observing, under these hypotheses, that $\left\langle x_{1}+A\right\rangle \oplus \cdots \oplus\left\langle x_{m}+A\right\rangle$ is at least a valuated coproduct in $G / A$. Indeed suppose that, for some choice of integers 
$t_{1}, \ldots, t_{m}$ and for some prime $p,\left(t_{1} x_{1}+\cdots+t_{m} x_{m}\right)+A$ is an element of $p^{\alpha}(G / A)$. By (3) there is a $k<\omega$ and a positive integer $n$ prime to $p$ such that $p^{k} n\left(t_{1} x_{1}+\cdots+t_{m} x_{m}\right)$ is in $p^{\alpha+k} G+A$, and therefore by (2) $n\left(t_{1} x_{1}+\cdots+t_{m} x_{m}\right)$ is in $p^{\alpha} G+A$. Since $A \oplus\left\langle x_{1}\right\rangle \oplus \cdots \oplus\left\langle x_{m}\right\rangle$ is a valuated coproduct and $n$ is prime to $p$, it follows that each $t_{i} x_{i}$ is in $p^{\alpha} G$ and a fortiori each $t_{i} x_{i}+A$ is in $p^{\alpha}(G / A)$. Next note that (3) has the following routine consequence: If $g+A$ is in $(G / A)\left(M^{*}, p\right)$, then there is some positive integer $n$, depending on $g$, such that $n g \in G\left(n M^{*}, p\right)+A$. From this and the fact that $A \oplus\left\langle x_{i}\right\rangle$ is a $*$-valuated coproduct, it quickly follows that each $x_{i}+A$ is a primitive element of $G / A$. Furthermore if $\left(t_{1} x_{1}+\cdots+t_{m} x_{m}\right)+A$ is in $(G / A)\left(M^{*}, p\right)$, then there is a positive integer $n$ such that $n\left(t_{1} x_{1}+\cdots+t_{m} x_{m}\right)$ belongs to $G\left(n M^{*}, p\right)+A$ and, because $A \oplus\left\langle x_{1}\right\rangle \oplus \cdots \oplus\left\langle x_{m}\right\rangle$ is a $*$-valuated coproduct, we have $n t_{i} x_{i}+A$ in $(G / A)\left(n M^{*}, p\right)$ for each $i=1, \ldots, m$. But since $\left\langle x_{1}+A\right\rangle \oplus \cdots \oplus\left\langle x_{m}+A\right\rangle$ is a valuated coproduct in $G / A$, we also have $t_{i} x_{i}+A \in(G / A)(M)$ for each $i$ and therefore, by an earlier observation, we actually have each $t_{i} x_{i}+A$ in $(G / A)\left(M^{*}, p\right)$, as desired. The corresponding verifications for subgroups of the form $(G / A)\left(M^{*}\right)$ and $(G / A)\left(\alpha^{*}, p\right)$ are similar, and hence we conclude that $\left\langle x_{1}+A\right\rangle \oplus \cdots \oplus\left\langle x_{m}+A\right\rangle$ is indeed a $*$-valuated coproduct.

Conversely, let us assume that $A$ is a subgroup of the mixed group $G$ with conditions (1), (2), and (3) satisfied. Then, given any finite subset $S$ of $G$, we need to exhibit primitive elements $x_{1}, \ldots, x_{m}$ such that $A^{\prime}=A \oplus\left\langle x_{1}\right\rangle \oplus \cdots \oplus$ $\left\langle x_{m}\right\rangle$ is a *-valuated coproduct in $G$ with $\left\langle S, A^{\prime}\right\rangle / A^{\prime}$ finite. But by (1) we at least have a direct sum $A^{\prime}=A \oplus\left\langle x_{1}\right\rangle \oplus \cdots \oplus\left\langle x_{n}\right\rangle$ such that $\left\langle S, A^{\prime}\right\rangle / A^{\prime}$ is finite, the elements $x_{i}+A$ are primitive in $G / A$ and $\left\langle x_{1}+A\right\rangle \oplus \cdots \oplus\left\langle x_{m}+A\right\rangle$ is a *valuated coproduct in the quotient group $G / A$. Since none of these conditions will be changed if the $x_{i}$ 's are replaced by nonzero multiplies of themselves, condition (3) allows us to assume that $\left\|x_{i}\right\|_{G}=\left\|x_{i}+A\right\|_{G / A}$ for $i=1, \ldots, m$. This last assumption quickly leads to the conclusion that each $x_{i}$ is a primitive element of $G$ (see, for example, the proof of Lemma 3.6 in [HM4]). It also yields the fact that $A \oplus\left\langle x_{1}\right\rangle \oplus \cdots \oplus\left\langle x_{m}\right\rangle$ is a valuated coproduct in $G$. Then suppose $a+t_{1} x_{1}+\cdots+t_{m} x_{m} \in G\left(M^{*}, p\right)$, where $a \in A$. We need to show that each $t_{i} x_{i}$ is in $G\left(M^{*}, p\right)$. But since $\left\langle x_{1}+A\right\rangle \oplus \cdots \oplus\left\langle x_{m}+A\right\rangle$ is a *-valuated coproduct, we at least have $t_{i} x_{i}+A \in(G / A)\left(M^{*}, p\right)$ for $i=1,2, \ldots, m$. By the primitivity of $x_{i}+A$, this implies that either $\left\|t_{i} x_{i}\right\|=\left\|t_{i} x_{i}+A\right\| \nsim M$ or $\left|p^{k} t_{i} x_{i}\right|_{p}=\left|p^{k} t_{i} x_{i}+A\right|_{p} \neq m_{p, k}$ for infinitely many values of $k<\omega$. In either case, $t_{i} x_{i} \in G\left(M^{*}, p\right)$, as desired. Once again, the verifications for subgroups of the form $G\left(M^{*}\right)$ and $G\left(\alpha^{*}, p\right)$ are similar, and thus $A \oplus\left\langle x_{1}\right\rangle \oplus \cdots \oplus\left\langle x_{m}\right\rangle$ is a $*$-valuated coproduct.

Remark 1.8. Let $A$ be a subgroup of the mixed group $G$ such that conditions (2) and (3) of Proposition 1.7 are satisfied. If $H \oplus\left\langle x_{1}\right\rangle \oplus \cdots \oplus\left\langle x_{m}\right\rangle$ is a *valuated coproduct in $G$ where the $x_{i}$ 's are primitive elements and $A \subseteq H$, then $H / A \oplus\left\langle x_{1}+A\right\rangle \oplus \cdots \oplus\left\langle x_{m}+A\right\rangle$ is a $*$-valuated coproduct and the $x_{i}+A$ 's are primitive in $G / A$. Indeed this follows from a slight modification of the first half of the proof of Proposition 1.7.

Following Warfield [W], we say that a short exact sequence $0 \rightarrow A \rightarrow B \rightarrow$ $C \rightarrow 0$ is sequentially pure if, for each height matrix $M$, the induced sequence $0 \rightarrow A(M) \rightarrow B(M) \rightarrow C(M) \rightarrow 0$ is also exact. A subgroup $H$ of a mixed group $G$ will be called a sequentially pure subgroup provided the canonical 
induced short exact sequence $0 \rightarrow H \rightarrow G \rightarrow G / H \rightarrow 0$ is sequentially pure. Clearly $H$ is sequentially pure in $G$ if and only if $H$ is isotype in $G$ and $(G / H)(M)=(G(M)+H) / H$ for every height matrix $M$.

Proposition 1.9. Let $H$ be a subgroup of the mixed group $G$. Then $H$ is isotype and knice in $G$ if and only if $G / H$ is a k-group and $H$ is sequentially pure in $G$.

Proof. Suppose $H$ is a sequentially pure subgroup of $G$. Then not only is $H$ isotype in $G$, but obviously $H$ is also a nice subgroup of $G$ since $p^{\alpha}(G / H)=$ $\left(p^{\alpha} G+H\right) / H$ for all primes $p$ and all ordinals $\alpha$. Thus if $G / H$ is also a $k$ group, then $H$ will be a knice subgroup of $G$ by Proposition 1.7. Conversely, let us assume that $H$ is both isotype and knice in $G$. That $G / H$ is a $k$-group follows from Proposition 1.7, and it remains only to show that $H$ is sequentially pure in $G$. Towards this end, suppose $g+H \in(G / H)(M)$ for some height matrix $M$. Then (3) of the preceding proposition tells us that there is a positive integer $n$ such that $n g \in G(n M)+H$. Since, however, $H \cap n G=n H$, we can write $g+h_{1}=y+g_{1}$ where $h_{1} \in H, y \in G(M)$ and $g_{1} \in G[n]$. But then $g_{1}+H \in(G / H)(M)$ and hence we may assume without loss of generality that $g$ has finite order. In fact, resolving $g$ into its primary components, we need only deal with the situation where $g+H \in(G / H)(M)$ and $p^{k} g=0$ for some prime $p$ and some $k<\omega$. In this special case, we show that $g=z+h$ where $h \in H$, $p^{k} z=0$, and $\left|p^{i} z\right|_{p} \geq m_{p, i}$ for $i<k$. We proceed by induction on $k$, and thus assume that the desired conclusion holds for $k-1$. Since $H$ is both nice and isotype in $G$, it is routine to see that $p^{\alpha}(G / H)[p]=\left(p^{\alpha} G[p]+H\right) / H$ for all ordinals $\alpha$. Consequently, $p^{k-1} g \in\left(p^{\alpha} G\right)[p]+H$, where $\alpha=m_{p, k-1}$ and it is easily seen that we can write $g=z_{0}+h_{0}+g^{\prime}$, where $\left|p^{i} z_{0}\right|_{p} \geq m_{p, i}$ for $i \leq k-1, p^{k} z_{0}=0, h_{0} \in H$, and $p^{k-1} g^{\prime}=0$. But then $g^{\prime}+H=g-z_{0}+H$ is in $(G / H)(M)$ and, by our inductive assumption, we have $g^{\prime}=z^{\prime}+h^{\prime}$, where $h^{\prime} \in H, p^{k-1} z^{\prime}=0$, and $\left|p^{i} z^{\prime}\right|_{p} \geq m_{p, i}$ for $i<k-1$. Taking $z=z_{0}+z^{\prime}$ and $h=h_{0}+h^{\prime}$, we have the desired representation of $g$.

Corollary 1.10. If the subgroup $H$ is both knice and pure in $G$, then $p^{\alpha}(G / H)=$ $\left(p^{\alpha} G+H\right) / H$ for all primes $p$ and all ordinals $\alpha$.

Proof. Let $g+H$ be an element of $p^{\alpha}(G / H)$. Exploiting the kniceness and purity of $H$, as in the preceding proof, we reduce considerations to the case where $g$ has finite order. Since $p^{\alpha} G$ contains all elements having finite order prime to $p$, we may further assume that $g$ has order a power of $p$. In this special case, the desired conclusion follows from the fact that $H$ is a nice subgroup of $G$.

\section{SUBGROUPS OF $k$-GROUPS}

In this section, we prove that any knice isotype subgroup of a $k$-group is itself a $k$-group. In particular, direct summands of $k$-groups are once again $k$-groups. Although not needed in subsequent parts of this paper, we include a proof that direct summands of groups with decomposition bases also have decomposition bases. In fact, the transition from the summand theorem for $k$-groups to the summand theorem for groups with decomposition bases is little more than an application of Kaplansky's classical result on summands of direct sums of countable groups. Our purely group-theoretical proof of this theorem 
may be welcomed as an alternative approach to the abstract proof in [AHR] via the global Azumaya theorem. (We warn the unwary that the purported group-theoretical proof of this result in $[\mathrm{S}]$ is seriously flawed.) The key to our approach is Theorem 2.3, which should remind the reader of the crucial step in Fuch's proof of the summand theorem for torsion-free separable groups [F2]. We begin with an ad hoc definition which proves to be quite convenient for our purposes.

Definition 2.1. A subgroup $C$ of the mixed group $G$ is said to be $k$-basic in $G$ provided it satisfies the following two conditions:

(1) If $C$ contains the primitive element $x$, then there is a $*$-valuated coproduct $C^{\prime}=\langle x\rangle \oplus D$ with $C / C^{\prime}$ finite.

(2) If $S$ is an arbitrary finite subset of $C$, then there are primitive elements $x_{1}, \ldots, x_{n}$ contained in $C$ such that $A=\left\langle x_{1}\right\rangle \oplus \cdots \oplus\left\langle x_{n}\right\rangle$ is a $*$-valuated coproduct with $\langle S, A\rangle / A$ finite.

Some useful elementary facts about subgroups that are $k$-basic are summarized in our next proposition.

Proposition 2.2. (1) If $C=\bigoplus_{i \in I}\left\langle x_{i}\right\rangle$ is a *-valuated coproduct with each $x_{i}$ a primitive element of the mixed group $G$, then $C$ is $k$-basic in $G$.

(2) If the subgroup $C$ is $k$-basic in $G$ and if $C / C^{\prime}$ is torsion, then $C^{\prime}$ is also $k$-basic in $G$.

(3) Let $C$ be $k$-basic in $G$ and suppose $C$ contains the $*$-valuated coproduct $\left\langle x_{1}\right\rangle \oplus \cdots \oplus\left\langle x_{n}\right\rangle$ where the $x_{i}$ 's are primitive elements. Then, for any finite subset $S$ of $C$, there exists primitive elements $y_{1}, \ldots, y_{m}$ such that $A=\left\langle x_{1}\right\rangle \oplus \cdots \oplus$ $\left\langle x_{n}\right\rangle \oplus\left\langle y_{1}\right\rangle \oplus \cdots \oplus\left\langle y_{m}\right\rangle$ is a $*$-valuated coproduct with $\langle S, A\rangle / A$ finite.

(4) If the subgroup $C$ is $k$-basic in $G$ and if $C^{\prime}=\left\langle x_{1}\right\rangle \oplus \cdots \oplus\left\langle x_{n}\right\rangle \oplus B$ is a *-valuated coproduct where $C / C^{\prime}$ is torsion and the $x_{i}$ 's are primitive elements, then $B$ is $k$-basic in $G$.

Proof. (1) Suppose $C=\bigoplus_{i \in I}\left\langle x_{i}\right\rangle$ is a $*$-valuated coproduct where the $x_{i}$ 's are primitive elements of $G$. That $C$ satisfies the first defining property for being $k$-basic follows from Proposition 1.3(4) with $A=0$, whereas, the second requisite property is trivial. Next observe that any subgroup of one that is $k$ basic will inherit the condition (1) of Definition 2.1. So in proving (2) and (4), we need only deal with the second condition of the definition. But in the case of (2), that second condition is an immediate consequence of the fact that a nonzero multiple of a primitive element is still primitive.

(3) Let $S^{\prime}=S \cup\left\{x_{1}, \ldots, x_{n}\right\}$ and choose primitive elements $z_{1}, \ldots, z_{k}$ in $C$ so that $A^{\prime}=\left\langle z_{1}\right\rangle \oplus \cdots \oplus\left\langle z_{k}\right\rangle$ is a $*$-valuated coproduct where $\left\langle S^{\prime}, A^{\prime}\right\rangle / A^{\prime}$ is finite. In particular, $A^{\prime}$ contains nonzero multiples $x_{1}^{\prime}, \ldots, x_{n}^{\prime}$ of $x_{1}, \ldots, x_{n}$, respectively. Then, by Corollary 1.4, there are primitive elements $y_{1}, \ldots, y_{m}$ such that $A=\left\langle x_{1}^{\prime}\right\rangle \oplus \cdots \oplus\left\langle x_{n}^{\prime}\right\rangle \oplus\left\langle y_{1}\right\rangle \oplus \cdots\left\langle y_{m}\right\rangle$ is a $*$-valuated coproduct with $A^{\prime} / A$ finite. Clearly $\langle S, A\rangle / A$ is finite and an application of the Seesaw Lemma, Proposition 2.7 in [HM4], allows us to replace the $x_{i}^{\prime}$ 's by the $x_{i}$ 's.

(4) As noted above, it suffices to show that $B$ satisfies part (2) of Definition 2.1. Let $W=\left\langle x_{1}\right\rangle \oplus \cdots \oplus\left\langle x_{n}\right\rangle$. Then by part (3) of this proposition, we have primitive elements $y_{1}, \ldots, y_{m}$ contained in $C$ such that $A=W \oplus\left\langle y_{1}\right\rangle \oplus \cdots \oplus$ $\left\langle y_{m}\right\rangle$ is a $*$-valuated coproduct with $\langle S, A\rangle / A$ finite. By replacing the $y_{i}$ 's by nonzero multiples of themselves, we may assume that they are all contained in 
$C^{\prime}=W \oplus B$. Thus for each $j$, we can write $y_{j}=w_{j}+b_{j}$, where $w_{j} \in W$ and $b_{j} \in B$. Because both $W \oplus B$ and $W \oplus\left\langle y_{1}\right\rangle \oplus \cdots \oplus\left\langle y_{m}\right\rangle$ are valuated coproducts, it follows that $\left\|y_{j}\right\|=\left\|b_{j}\right\|$ for all $j$. Hence an application of Proposition 1.3(2) shows that $A=W \oplus\left\langle b_{1}\right\rangle \oplus \cdots \oplus\left\langle b_{m}\right\rangle$ is a $*$-valuated coproduct with the $b_{j}$ 's primitive elements. Since $\langle S\rangle \subseteq B,\left\langle S, B^{\prime}\right\rangle / B^{\prime}$ is finite where $B^{\prime}=\left\langle b_{1}\right\rangle \oplus \cdots \oplus\left\langle b_{m}\right\rangle$ is the required $*$-valuated coproduct contained in $B$.

Theorem 2.3. Suppose $C=A \oplus B$ is a $*$-valuated coproduct where the subgroup $C$ is $k$-basic in the group $G$ and let $x$ be a primitive element contained in $C$. Then there is a *-valuated coproduct $C^{\prime}=\left\langle x^{\prime}\right\rangle \oplus\langle y\rangle \oplus A^{\prime} \oplus B^{\prime}$ where $C / C^{\prime}$ is finite, $A^{\prime} \subseteq A, B^{\prime} \subseteq B, x^{\prime}$ is a nonzero multiple of $x$ and either $y=0$ or else $y$ is a primitive element with $\|y\| \sim\|x\|$.

Proof. Write $x=a_{0}+b_{0}$, where $a_{0} \in A, b_{0} \in B$ and let $M=\|x\|$. Since $C$ is $k$-basic in $G$, there is a positive integer $\delta$ such that $\delta a_{0}=r x+x_{2}+\cdots+x_{m}+w_{0}$ where $\langle x\rangle \oplus\left\langle x_{2}\right\rangle \oplus \cdots \oplus\left\langle x_{m}\right\rangle \oplus\left\langle w_{0}\right\rangle$ is a $*$-valuated coproduct, each $x_{i}$ is a primitive element contained in $C$ with $\left\|x_{i}\right\| \sim M$ and $w_{0} \in G\left(M^{*}\right)$. Then $\delta b_{0}=s x-\left(x_{2}+\cdots+x_{m}+w_{0}\right)$ where $s=\delta-r$. We first reduce consideration to the case where $r \neq 0$ and $s \neq 0$. If, for example, $s=0$, then $\delta a_{0}=$ $\delta x+x_{2}+\cdots+x_{m}+w_{0}$ and it follows, because of the two valuated coproducts involved, that $\left\|\delta a_{0}\right\|=\|\delta x\|$. Then, by Proposition 1.3(2), $\delta a_{0}$ is primitive and therefore, since $C$ is $k$-basic, there is a $*$-valuated coproduct $C_{1}=\left\langle\delta a_{0}\right\rangle \oplus C_{2}$ with $C / C_{1}$ finite. Thus we have *-valuated coproducts $A \cap C_{1}=\left\langle\delta a_{0}\right\rangle \oplus A^{\prime}$ and $C^{\prime}=\left\langle\delta a_{0}\right\rangle \oplus A^{\prime} \oplus B$ where $C / C^{\prime}$ is finite because $A / A \cap C_{1} \simeq\left(A+C_{1}\right) / C_{1}$ is. From Proposition 1.3(2) again and the equation $\delta x=\delta a_{0}+\delta b_{0}$, it follows that $C^{\prime}=\langle\delta x\rangle \oplus A^{\prime} \oplus B$, as desired. The case where $r=0$ is, of course, similar.

In the more general situation where neither $r$ nor $s$ vanishes, neither $a_{0}$ nor $b_{0}$ will necessarily be primitive. Nonetheless we shall show that it is possible to extract primitive elements $a$ and $b$ from $A$ and $B$, respectively, such that $\left\|x^{\prime}\right\|=\left\|a^{\prime}+b^{\prime}\right\|$ where the primed quantities are appropriate nonzero multiples of the corresponding unprimed quantities. The primitive elements $a$ and $b$ will determine the complements $A^{\prime} \subseteq A$ and $B^{\prime} \subseteq B$, and Proposition 1.3 will be applied to obtain the desired decomposition of the subgroup $C^{\prime}$. Towards this end, let $x_{1}=r x$ and, for $i=1,2, \ldots, m$, write $x_{i}=a_{i}+b_{i}$ where $a_{i} \in A$ and $b_{i} \in B$. Exploiting again the fact that $C$ is $k$-basic in $G$ (see Proposition 2.2(3)), we form a *-valuated coproduct $F=\left\langle x_{1}\right\rangle \oplus \cdots \oplus\left\langle x_{n}\right\rangle \oplus W$ in $C$ where $n \geq m$, each $x_{i}$ is primitive with $\left\|x_{i}\right\| \sim M, W$ is generated by primitive elements not quasi-equivalent to $M$, and there is a fixed positive integer $k$ such that $k a_{i} \in F$ for $i=1,2, \ldots, m$. Since $M \leq\left\|x_{i}\right\| \leq\left\|a_{i}\right\|$ whenever $i \leq m$, we may restrict $W$ so that it is contained in $G\left(M^{*}\right)$ and furthermore, replacing $x$ by a multiple if necessary, we may assume also that $w_{0} \in W$. We now write for each $i \leq m$,

$$
k a_{i}=\sum_{j=1}^{n} c_{i j} x_{j}+w_{i},
$$

where each $w_{i}$ is in $W$ and the $c_{i j}$ 's are integers. Now note that $b_{1}+\cdots+b_{m}$, the $B$-component of $x_{1}+\cdots+x_{m}=\delta a_{0}-w_{0}$, necessarily lies in $G\left(M^{*}\right) \cap F=W$ and therefore $k\left(x_{1}+\cdots+x_{m}\right)=\sum_{i=1}^{m} \sum_{j=1}^{n} c_{i j} x_{j}+w$ for some $w \in W$. Comparing coefficients of $x_{j}$ on either side of the equation, we conclude that

$$
k=c_{1 j}+c_{2 j}+\cdots+c_{m j}
$$


holds for each $j=1,2, \ldots, m$. The preceding equation will play an important role in selecting the desired primitive element $a \in A$.

Since we are free to replace $x$ by any nonzero multiple of itself, there is no loss of generality in assuming that $(\delta, r)=1$ and consequently that $(r, s)=$ 1. For technical reasons, it is more convenient to construct an appropriate primitive element $g$ in $C$ having the desired $a$ as its $A$-component rather than constructing $a$ directly. This construction will require a fixed finite set $\Lambda$ of primes that do not divide $r$, with $\Lambda$ being precisely specified later. The primitive element $g$ will be chosen so as to satisfy the following condition:

$$
g=t\left(\delta a_{0}-w_{0}\right)+g^{\prime}=c_{1} x_{1}+c_{2} x_{2}+\cdots+c_{n} x_{n},
$$

where $g^{\prime} \in A+W$ and, for all primes $p \in \Lambda$, we have that $(t, p)=1$, $\left\|g^{\prime}\right\|_{p} \geq\|p g\|_{p}$, and $\|g\|_{p}=\left\|\delta a_{0}\right\|_{p}=\|x\|_{p}=\left\|c_{1} x_{1}\right\|_{p}$.

Observe that condition (3) is satisfied if we simply take $g=\delta a_{0}-w_{0}=$ $x_{1}+x_{2}+\cdots+x_{m}$, though this particular $g$ need not necessarily be primitive. To find a primitive $g$ satisfying (3) it is helpful to introduce the set $P$ consisting of all those primes $p$ for which there exists some $\alpha<\omega$ such that $\left\|p^{\alpha} x_{1}\right\|_{p}=$ $\left\|p^{\alpha} x_{2}\right\|_{p}=\cdots=\left\|p^{\alpha} x_{n}\right\|_{p}$. Then for any element $g$ satisfying (3), we take $Q(g)$ to consist of all those primes $p \notin P \backslash \Lambda$ for which there exists an $i \leq n$ such that $\|g\|_{p}=\left\|c_{i} x_{i}\right\|_{p}$. Since $P$ is cofinite in $\mathbf{P}, Q(g)$ is a finite set of primes containing $\Lambda$. Moreover, by Proposition 1.3(3), $g$ will be primitive provided $Q(g) \cup P=\mathbf{P}$. Therefore we may conclude that the desired primitive element exists if we can show that whenever $q$ is a prime not in $Q(g) \cup P$, there will be a $\bar{g}$ satisfying (3) and such that $Q(\bar{g}) \supseteq Q(g) \cup\{q\}$.

So suppose $g$ satisfies (3) and that $q$ is a fixed prime with $q \notin Q(g) \cup P$. A little reflection leads to the conclusion that there is a nonempty subset $J$ of $\{1,2, \ldots, m\}$ and a $\gamma<\omega$ such that $\left\|q^{\gamma} x_{l}\right\|_{q}=\left\|q^{\gamma} x_{r}\right\|_{q}$ for all $l, r \in J$, and

$$
\begin{array}{ll}
\left|q^{\alpha} x_{i}\right|_{q}>\left|q^{\alpha} x_{l}\right|_{q} \neq \infty & \text { for infinitely many } \alpha<\omega \\
& \text { whenever } l \in J, i \leq m, \text { and } i \notin J .
\end{array}
$$

Furthermore, replacing $x$ by $q^{\gamma} x$, we may assume without loss of generality that $\left\|x_{l}\right\|_{q}=\left\|x_{r}\right\|_{q}$ for all $l, r \in J$. Then write $k=q^{j} k^{\prime}$, where $\left(k^{\prime}, q\right)=1$. We first note that $q^{j+1} \mid c_{i l}$ whenever $l \in J, i \leq m$, and $i \notin J$. Indeed if $q^{j+1} \nmid c_{i l}$, then we would have

$$
\left|q^{\alpha+j} x_{l}\right|_{q} \geq\left|q^{\alpha} c_{i l} x_{l}\right|_{q} \geq\left|q^{\alpha} k a_{i}\right|_{q}=\left|q^{\alpha+j} a_{i}\right| \geq\left|q^{\alpha+j} x_{i}\right|_{q}
$$

for all $\alpha<\omega$; and this would contradict (4). Next observe that we have $q^{j} \mid c_{l r}$ whenever $l, r \in J$ because

$$
\left\|k a_{l}\right\|_{q} \leq\left\|c_{l r} x_{r}\right\|_{q}=\left\|c_{l r} x_{l}\right\|_{q} \leq\left\|c_{l r} a_{l}\right\|_{q} .
$$

On the other hand, for a fixed $r \in J$, (2) implies that there must exist some $l \in J$ such that $q^{j+1} \nmid c_{l r}$. But for this choice of $l, r \in J$, we have $\left\|q^{j} x_{l}\right\|_{q}=$ $\left\|k x_{l}\right\|_{q} \leq\left\|k a_{l}\right\|_{q} \leq\left\|c_{l r} x_{r}\right\|_{q}=\left\|q^{j} x_{r}\right\|_{q}=\left\|q^{j} x_{l}\right\|_{q}$, that is,

$$
\left\|k x_{l}\right\|_{q}=\left\|k a_{l}\right\|_{q}=\left\|k a_{l}-w_{l}\right\|_{q}=\left\|c_{l r} x_{r}\right\|_{q} .
$$

Exploiting the fact that $\left\|k a_{l}-w_{l}\right\| \sim\|g\|$, we choose positive integers $c$ and $\beta$ such that $(c, q)=1$ and the following two conditions hold:

$$
\begin{gathered}
\left\|q^{\beta} g\right\|_{q} \geq\left\|q\left(k a_{l}-w_{l}\right)\right\|_{q} . \\
\left\|c\left(k a_{l}-w_{l}\right)\right\|_{p} \geq\|p g\|_{p} \quad \text { for all } p \in Q(g) .
\end{gathered}
$$


Now let $\bar{g}=q^{\beta} g+c\left(k a_{l}-w_{l}\right)=\bar{c}_{1} x_{1}+\bar{c}_{2} x_{2}+\cdots+\bar{c}_{n} x_{n}$, where $\bar{c}_{i}=q^{\beta} c_{i}+c c_{l i}$ for $i=1,2, \ldots, n$. Since $(c, q)=1$ and $\left\|c_{r} x_{r}\right\| \geq\|g\|$, it follows from (5) and (6) that

$$
\|\bar{g}\|_{q}=\left\|k a_{l}-w_{l}\right\|_{q}=\left\|c_{l r} x_{r}\right\|_{q}=\left\|\bar{c}_{r} x_{r}\right\|_{q} .
$$

On the other hand, if $p \in Q(g)$ with $\|g\|_{p}=\left\|c_{i} x_{i}\right\|_{p}$, then (7) and $\left\|c_{l i} x_{i}\right\| \geq$ $\left\|k a_{l}-w_{l}\right\|$ together imply that

$$
\|\bar{g}\|_{p}=\left\|q^{\beta} g\right\|_{p}=\left\|q^{\beta} c_{i} x_{i}\right\|_{p}=\left\|\bar{c}_{i} x_{i}\right\|_{p} .
$$

Furthermore, $\bar{g}^{\prime}=q^{\beta} g^{\prime}+c\left(k a_{l}-w_{l}\right)$ is in $A+W$ and if $p \in \Lambda$, then (7) yields

$$
\left\|\bar{g}^{\prime}\right\|_{p} \geq\left\|q^{\beta} g^{\prime}\right\|_{p} \wedge\left\|c\left(k a_{l}-w_{l}\right)\right\|_{p} \geq\|p g\|_{p}=\|p \bar{g}\|_{p} .
$$

From (8), (9) and (10), we conclude that $\bar{g}$ satisfies (3) and $Q(\bar{g}) \supseteq Q(g) \cup\{q\}$. This completes the proof of the existence of a primitive element $g$ as in (3). By symmetry, given a finite set $\Lambda^{\prime}$ of primes not dividing $s$, we see upon taking $x_{1}^{\prime}=s x$ that there is a primitive element $h$ in $C$ satisfying the following condition

$$
h=t^{\prime}\left(\delta b_{0}+w_{0}\right)+h^{\prime}=c_{1}^{\prime} x_{1}^{\prime}+c_{2}^{\prime} x_{2}+\cdots+c_{n}^{\prime} x_{n},
$$

where $h^{\prime} \in B+W$ and, for all primes $p \in \Lambda^{\prime}$, we have that $\left(t^{\prime}, p\right)=1$, $\left\|h^{\prime}\right\|_{p} \geq\|p h\|_{p}$, and $\|h\|_{p}=\left\|\delta b_{0}\right\|_{p}=\|x\|_{p}=\left\|c_{1}^{\prime} x_{1}^{\prime}\right\|_{p}$.

Since $C$ is $k$-basic in $G$ we have a $*$-valuated coproduct $C_{1}=\langle g\rangle \oplus D_{1}$ with $C / C_{1}$ finite. Therefore there will exist integers $d \neq 0$ and $\lambda$ such that

$$
d \delta a_{0}=\lambda g+u, \quad \text { where } u \in D_{1} .
$$

Replacing $x$ by a nonzero multiple of itself, we may also assume that $(d, \lambda)=1$ and $\omega_{0} \in D_{1}$. A routine calculation shows that

$$
t \lambda g+d g^{\prime}=d g+t\left(d \omega_{0}-u\right) \text {. }
$$

If $p \in \Lambda$ and $p \mid \lambda$, then (3), (12) and the fact that $\langle g\rangle \oplus D_{1}$ is a valuated coproduct imply that

$$
\|x\|_{p}=\|g\|_{p}=\|d g\|_{p} \geq\|t \lambda g\|_{p} \wedge\left\|d g^{\prime}\right\|_{p} \geq\|p g\|_{p}=\|p x\|_{p},
$$

that is, $|x|_{p}=\infty$. Hence we conclude that

$$
\text { if } p \in \Lambda \text { and }|x|_{p} \neq \infty \text {, then } p \nmid \lambda \text { and }\|x\|_{p}=\|g\|_{p} \text {. }
$$

Similarly, we have a $*$-valuated coproduct $C_{2}=\langle h\rangle \oplus D_{2}$ with $C / C_{2}$ finite and relatively prime integers $e \neq 0$ and $\mu$ such that

$$
e \delta b_{0}=\mu h+v, \quad \text { where } v \in D_{2},
$$

and

$$
\text { if } p \in \Lambda^{\prime} \text { and }|x|_{p} \neq \infty \text {, then } p \nmid \mu \text { and }\|x\|_{p}=\|h\|_{p} \text {. }
$$

We now specify $\Lambda$ and $\Lambda^{\prime}$ by taking $\Lambda=\{p \in \mathbf{P}: p \mid s\}$ and $\Lambda^{\prime}=\{p \in$ P: $\|g\|_{p} \neq\|x\|_{p}$ or $\left.p \mid \lambda\right\} \cap\left\{p \in \mathbf{P}:|x|_{p} \neq \infty\right\}$. Notice that, since $(r, s)=1$, the primes in $\Lambda$ do not divide $r$ and that $\Lambda^{\prime}$ is finite because $\|g\| \sim\|x\|$. Clearly 
(13) implies that $\Lambda \cap \Lambda^{\prime}=\varnothing$ and consequently primes in $\Lambda^{\prime}$ do not divide $s$. Next we establish the following crucial fact:

$$
\|d e \delta x\|_{p}= \begin{cases}\|e \lambda g\|_{p} & \text { if } p \notin \Lambda^{\prime} \\ \|d \mu h\|_{p} & \text { if } p \in \Lambda^{\prime} .\end{cases}
$$

Since $\|x\|=\left\|a_{0}\right\| \wedge\left\|b_{0}\right\|,(11)$ and $\left(11^{\prime}\right)$ imply that $\|d e \delta x\| \leq\|e \lambda g\| \wedge\|d \mu h\|$. Therefore (14) certainly holds for any prime $p$ such that $|x|_{p}=\infty$. If $p \in \Lambda^{\prime}$ and $|x|_{p} \neq \infty$, then it follows from $\left(13^{\prime}\right)$ that

$$
\|d e \delta x\|_{p} \leq\|d \mu h\|_{p}=\|d x\|_{p} \leq\|d e \delta x\|_{p} .
$$

On the other hand, if $p \notin \Lambda^{\prime}$ and $|x|_{p} \neq \infty$, then $\|g\|_{p}=\|x\|_{p}, p \nmid \lambda$ and

$$
\|d e \delta x\|_{p} \leq\|e \lambda g\|_{p}=\|e x\|_{p} \leq\|d e \delta x\|_{p} .
$$

Thus (14) does indeed hold in all cases.

Our next problem is to prove that $g$ can effectively be replaced by its $A$ component. Write $g=a+b^{\prime}$, where $a \in A$ and $b^{\prime} \in B$. By (3), $g \in A+W$ and consequently $b^{\prime}$ is in $G\left(M^{*}\right)$. In fact, we can show that $b^{\prime} \in G\left(\|g\|^{*}\right)$. Indeed since $C$ is $k$-basic, replacing $x$ by an appropriate nonzero multiple, we can write $b^{\prime}=y_{1}+\cdots+y_{j}$ where the $y_{i}$ 's are primitive and $\left\langle y_{1}\right\rangle \oplus \cdots \oplus\left\langle y_{j}\right\rangle$ is a $*$-valuated coproduct. Since each $y_{i}$ is a primitive element contained in $G\left(M^{*}\right),\left\|y_{i}\right\| \nsim M$. Finally $\|g\| \leq\left\|b^{\prime}\right\| \leq\left\|y_{i}\right\|$ and $\left\|y_{i}\right\| \nsim\|g\|$ imply that $y_{i} \in G\left(\|g\|^{*}\right)$ for each $i$, that is, $b^{\prime} \in G\left(\|g\|^{*}\right)$ as claimed. But then Proposition 1.3 (1) implies that $a$ is primitive and also that $\|a\|=\|g\|$ if $x$ is replaced by a suitable nonzero multiple of itself. Similarly, if $h=a^{\prime}+b$ where $a^{\prime} \in A$ and $b \in B$, then we may assume that $b$ is a primitive element with $\|b\|=\|h\|$. By once again replacing $x$ by a nonzero multiple, we may further assume that $b^{\prime} \in C_{1}=\langle g\rangle \oplus D_{1}$ and $a^{\prime} \in C_{2}=\langle h\rangle \oplus D_{2}$. Since, however, no nonzero multiple of $g$ or $h$ can lie in $G\left(M^{*}\right)$, we actually have $b^{\prime} \in D_{1}$ and $a^{\prime} \in D_{2}$. Therefore, by Proposition 1.3(2), $C_{1}=\langle a\rangle \oplus D_{1}$ and $C_{2}=\langle b\rangle \oplus D_{2}$. A routine argument shows that if $A^{\prime}=A \cap D_{1}$ and $B^{\prime}=B \cap D_{2}$, then $C_{0}=\langle a\rangle \oplus\langle b\rangle \oplus A^{\prime} \oplus B^{\prime}$ is a $*$-valuated coproduct with $C / C_{0}$ finite. Moreover, by $(11)$ and $\left(11^{\prime}\right)$, it follows that $d \delta a_{0}=\lambda a+u^{\prime}$ and $e \delta b_{0}=\mu b+v^{\prime}$, where $u^{\prime} \in A^{\prime}$ and $v^{\prime} \in B^{\prime}$. Thus

$$
d e \delta x=(e \lambda a+d \mu b)+\left(e u^{\prime}+d v^{\prime}\right),
$$

and (14) implies that $\|d e \delta x\|=\|e \lambda a+d \mu b\|$. But then, by Proposition 1.3(3), $z=e \lambda a+d \mu b$ is primitive and consequently Proposition 1.3(4) yields a primitive element $y \in\langle a, b\rangle$ such that $C^{\prime}=\langle z\rangle \oplus\langle y\rangle \oplus A^{\prime} \oplus B^{\prime}$ is a $*$-valuated coproduct with $C / C^{\prime}$ finite. One further application of Proposition 1.3(2) gives us the desired $*$-valuated coproduct $C^{\prime}=\left\langle x^{\prime}\right\rangle \oplus\langle y\rangle \oplus A^{\prime} \oplus B^{\prime}$ where $x^{\prime}=d e \delta x$ is a nonzero multiple of $x$.

Corollary 2.4. Suppose $C=A \oplus B$ is a *-valuated coproduct where $C$ is $k$ basic in the mixed group $G$ and $A$ has finite rank. Then there is a finite rank *-valuated coproduct $A^{\prime}=\left\langle z_{1}\right\rangle \oplus \cdots \oplus\left\langle z_{n}\right\rangle$ such that each $z_{i}$ is a primitive element and $A / A^{\prime}$ is torsion.

Proof. The proof is by induction on the rank of $A$, and we may take $A^{\prime}=0$ when $A$ has zero rank. When $A$ is not torsion, the finiteness of its rank insures that the maximum condition is satisfied for the quasi-equivalence classes realized by the elements of $A$. Consequently, $A$ contains an element $a_{0}$ of 
infinite order such that $A \cap G\left(M^{*}\right)=0$ where $M=\left\|a_{0}\right\|$. Since $C$ is a $k$ basic in $G$, there is a positive integer $\delta$ such that $\delta a_{0}=x_{1}+\cdots+x_{m}+w_{0}$ where $\left\langle x_{1}\right\rangle \oplus \cdots \oplus\left\langle x_{m}\right\rangle \oplus\left\langle w_{0}\right\rangle$ is a $*$-valuated coproduct, each $x_{i}$ is a primitive element contained in $C$ with $\left\|x_{i}\right\| \sim M$, and $w_{0} \in G\left(M^{*}\right)$. Taking $\Lambda=\varnothing$, the construction used in the proof of Theorem 2.3 yields a primitive element $g \in A+G\left(M^{*}\right)$ with $\|g\| \sim M$. Then, as in the preceding proof, if $g=a+b^{\prime}$ with $a \in A$ and $b^{\prime} \in B, a$ is also primitive with $\|a\| \sim M$. Because $C$ is $k$-basic in $G$, we have a $*$-valuated coproduct $C_{1}=\langle a\rangle \oplus D_{1}$ with $C / C_{1}$ finite. Then clearly $C^{\prime}=\langle a\rangle \oplus A^{\prime} \oplus B$ is a $*$-valuated coproduct with $C / C^{\prime}$ finite if we take $A^{\prime}=A \cap D_{1}$. Since $C^{\prime}$ is $k$-basic in $G$ by Proposition 2.2 (2), and rank $\left(A^{\prime}\right)<\operatorname{rank}(A)$, the desired conclusion follows by induction.

If $H \oplus K$ is a $*$-valuated coproduct in $G$, then we say that the subgroup $C$ is quasi-splitting along $H$ and $K$ provided each element of $C$ has finite order modulo $(C \cap H) \oplus(C \cap K)$.

Corollary 2.5. Suppose both $H \oplus K$ and $C=\bigoplus_{i=1}^{\infty}\left\langle x_{i}\right\rangle$ are $*$-valuated coproducts in $G$ where the $x_{i}$ 's are primitive and $C$ is quasi-splitting along $H$ and $K$. Then for each positive integer $n$, there is a $*$-valuated coproduct $C_{n}=\left\langle y_{1}\right\rangle \oplus$ $\cdots \oplus\left\langle y_{n}\right\rangle \oplus A_{n} \oplus B_{n}$ where $C / C_{n}$ is torsion, $A_{n} \subseteq H, \operatorname{rank}\left((C \cap H) / A_{n}\right) \leq n$ and the elements $y_{1}, \ldots, y_{n}$ are nonzero multiples of $x_{1}, \ldots, x_{n}$, respectively. Proof. First observe in Theorem 2.3 that the quotient group $A / A^{\prime}$ has rank at most 1. By Proposition 2.2, $C^{\prime}=(C \cap H) \oplus(C \cap K)$ is $k$-basic in $G$. Applying Theorem 2.3, we have a $*$-valuated coproduct $C_{1}=\left\langle y_{1}\right\rangle \oplus A_{1} \oplus B_{1}$ where $C^{\prime} / C_{1}$ is finite, $y_{1}$ is a nonzero multiple of $x_{1}, A_{1} \subseteq C \cap H$, and $\operatorname{rank}\left((C \cap H) / A_{1}\right) \leq$ 1. Thus the desired result holds for $n=1$. Assume that Corollary 2.5 holds for some integer $n-1 \geq 1$, and let $C_{n-1}=\left\langle y_{1}\right\rangle \oplus \cdots \oplus\left\langle y_{n-1}\right\rangle \oplus A_{n-1} \oplus B_{n-1}$ be a $*$-valuated coproduct with the requisite properties. Since $C / C_{n-1}$ is torsion, some nonzero multiple $y_{n}$ of $x_{n}$ lies in $C_{n-1}$, say, $y_{n}=z+a+b$, where $z \in\left\langle y_{1}, \ldots, y_{n-1}\right\rangle, a \in A_{n-1}$, and $b \in B_{n-1}$. Note that $x=y_{n}-z$ lies in $D=A_{n-1} \oplus B_{n-1}$ and that $\|x\|=\left\|y_{n}\right\| \wedge\|z\|=\left\|y_{n}\right\|$. By Proposition 1.3(2) and Proposition 2.2(4), $x$ is primitive and $D$ is $k$-basic in $G$. Replacing $x$ and $y_{n}$ by nonzero multiples and applying Theorem 2.3 again, we may assume that we have a $*$-valuated coproduct $D^{\prime}=\langle x\rangle \oplus A_{n} \oplus B_{n}$ where $D / D^{\prime}$ is finite, $A_{n} \subseteq A_{n-1}$, and $\operatorname{rank}\left(A_{n-1} / A_{n}\right) \leq 1$. Another application of Proposition 1.3(2) yields the $*$-valuated coproduct $C_{n}=\left\langle y_{1}\right\rangle \oplus \cdots \oplus\left\langle y_{n-1}\right\rangle \oplus\left\langle y_{n}\right\rangle \oplus A_{n} \oplus B_{n}$. A routine argument shows that $C / C_{n}$ is torsion and that $\operatorname{rank}\left((C \cap H) / A_{n}\right) \leq$ $1+\operatorname{rank}\left((C \cap H) / A_{n-1}\right) \leq n$.

Theorem 2.6. A direct summand of a $k$-group is itself a $k$-group.

Proof. Suppose $G=H \oplus K$ is a $k$-group and let $S$ be an arbitrary finite subset of $H$. Since $G$ is a $k$-group, there are primitive elements $x_{1}, \ldots, x_{n}$ in $G$ such that $F=\left\langle x_{1}\right\rangle \oplus \cdots \oplus\left\langle x_{n}\right\rangle$ is a $*$-valuated coproduct with $\langle S, F\rangle / F$ finite. By repeated applications of Proposition 1.6(2), $F$ can be enlarged to a $*$-valuated coproduct $C=\bigoplus_{i=1}^{\infty}\left\langle x_{i}\right\rangle$, where all the $x_{i}$ 's are primitive and $C$ is quasi-splitting along $H$ and $K$. (For the details of this construction, see the proof of Theorem 3.7 in [HM3].) By Corollary 2.5, we have nonzero multiples $y_{1}, \ldots, y_{n}$ of $x_{1}, \ldots, x_{n}$, respectively, and a $*$-valuated coproduct $C_{n}=F^{\prime} \oplus A_{n} \oplus B_{n}$, where $C / C_{n}$ is torsion, $A_{n} \subseteq H, \operatorname{rank}\left((C \cap H) / A_{n}\right) \leq n$, and $F^{\prime}=\left\langle y_{1}, \ldots, y_{n}\right\rangle$. Clearly $\left\langle S, F^{\prime}\right\rangle / F^{\prime}$ is also finite. Observe then that $C_{n} \cap H=A_{n} \oplus A$ where $A=H \cap\left(F^{\prime} \oplus B_{n}\right)$. Moreover, $\langle S, A\rangle / A$ is finite 
since $S \subseteq H$, and $\operatorname{rank}(A) \leq n$ because $A \cong\left(C_{n} \cap H\right) / A_{n}$. Notice that $C_{n}$ too is quasi-splitting along $H$ and $K$ because $C / C_{n}$ is finite, and therefore $C^{\prime}=\left(C_{n} \cap H\right) \oplus\left(C_{n} \cap K\right)=A_{n} \oplus A \oplus\left(C_{n} \cap K\right)$ is $k$-basic in $G$ by Proposition 2.2(2). Thus Corollary 2.4 is applicable to yield a $*$-valuated coproduct $A^{\prime}=$ $\left\langle z_{1}\right\rangle \oplus \cdots \oplus\left\langle z_{m}\right\rangle$, where the $z_{i}$ 's are primitive and $A / A^{\prime}$ is torsion. Since $H$ is a direct summand of $G$, the elements $z_{i}$ are primitive as elements of $H$ and $A^{\prime}=\left\langle z_{1}\right\rangle \oplus \cdots \oplus\left\langle z_{m}\right\rangle$ is a $*$-valuated coproduct in $H$. Clearly $\left\langle S, A^{\prime}\right\rangle / A^{\prime}$ is still finite and we conclude that $H$ is a $k$-group.

Corollary 2.7. A direct summand of $k$-group is a knice subgroup.

Proof. Suppose $G=H \oplus K$ is a $k$-group. That $H$ is knice subgroup of $G$ is an easy consequence of the fact that the complementary summand $K$ is a $k$-group.

In view of the preceding corollary, our next result may be viewed as a generalization of Theorem 2.6.

Theorem 2.8. If $H$ is a knice isotype subgroup of the $k$-group $G$, then $H$ is itself a $k$-group.

Proof. Let $S$ be a finite subset of $H$ and choose $F=\left\langle x_{1}\right\rangle \oplus \cdots \oplus\left\langle x_{n}\right\rangle$ exactly as in the proof of Theorem 2.6. In order to apply the same argument as in the earlier proof we require *-valuated coproducts $H \oplus K$ and $C=\bigoplus_{i=1}^{\infty}\left\langle x_{i}\right\rangle$ where the $x_{i}$ 's are primitive and $C$ is quasi-splitting along $H$ and $K$. Such a $C$ and $K$ can be obtained by repeated applications of Proposition 1.6 (2); and indeed the details of this construction are essentially as in the proof of Theorem 4.8 in [HM3]. Then just as in the proof of Theorem 2.6 there is a *-valuated coproduct $A^{\prime}=\left\langle z_{1}\right\rangle \oplus \cdots \oplus\left\langle z_{m}\right\rangle \subseteq H$ where the $z_{i}$ 's are primitive and $\left\langle S, A^{\prime}\right\rangle / A^{\prime}$ is finite. That the $z_{i}$ 's are primitive as elements of $H$ follows from the fact that $H$ is isotype in $G$ (see Definition 1.1), and the fact that $A^{\prime}=\left\langle z_{1}\right\rangle \oplus \cdots \oplus\left\langle z_{m}\right\rangle$ is still a $*$-valuated coproduct when viewed as a subgroup of $H$ depends on the primitivity of the $z_{i}$ 's.

Remark 2.9. Not surprisingly, the foregoing developments imply the following: If $H \oplus K$ is a *-valuated coproduct in the mixed group $G$ and if $H \oplus K$ is $k$-basic in $G$, then the subgroup $H$ is also $k$-basic in $G$. As noted before, any subgroup of $H \oplus K$ will inherit condition (1) of Definition 2.1. Thus it remains to show that each finite subset $S$ of $H$ is finite modulo a *-valuated coproduct $\left\langle z_{1}\right\rangle \oplus \cdots \oplus\left\langle z_{m}\right\rangle$ where the $z_{i}$ 's are primitive elements contained in $H$. But this is substantially established in the proof of Theorem 2.6 since the fact that $H \oplus K$ is $k$-basic in $G$ is enough to allow, within the subgroup $H \oplus K$, the construction of subgroups $F$ and $C$ with the requisite properties.

Theorem 2.10. If the mixed group $G$ contains a decomposition basis, then so does any direct summand of $G$.

Proof. Let $G=H \oplus K$ and suppose $X$ is a decomposition basis for $G$. We may assume that $X$ is infinite since the finite rank case is covered by Theorem 2.6. Let $A=\langle X\rangle$ and take $A_{0}=0$. By a straightforward back-and-forth argument, $A$ is the union of a smooth chain $\left(A_{\alpha}\right)_{\alpha<\mu}$ where each $A_{\alpha}$ is generated by a subset of $X$ and is quasi-splitting along $H$ and $K$, and furthermore $A_{\alpha+1} / A_{\alpha}$ is countable for each $\alpha<\mu$. The chief problem in completing the proof is to exhibit, for each $\alpha$, a countable subgroup $C_{\alpha}$ of $H$ such that 
(i) $C_{\alpha}$ is $k$-basic in $G$,

(ii) $\left(A_{\alpha} \cap H\right) \oplus C_{\alpha}$ is a $*$-valuated coproduct, and

(iii) $\left(A_{\alpha+1} \cap H\right) /\left(\left(A_{\alpha} \cap H\right) \oplus C_{\alpha}\right)$ is torsion.

If such $C_{\alpha}$ 's can be found, then clearly $C=\bigoplus_{\alpha<\mu} C_{\alpha}$ will be a *-valuated coproduct with $H / C$ torsion. But since each $C_{\alpha}$ is countable and $k$-basic in $G$, repeated applications of Proposition 2.2(3) insure that there exists a collection $\left(x_{i}\right)_{i \in I_{\alpha}}$ of primitive elements such that $B_{\alpha}=\bigoplus_{i \in I_{\alpha}}\left\langle x_{i}\right\rangle$ is a *valuated coproduct with $C_{\alpha} / B_{\alpha}$ torsion. Therefore, taking $I=\bigcup_{\alpha<\mu} I_{\alpha},\left(x_{i}\right)_{i \in I}$ is a decomposition bases of $H$.

To complete the proof of Theorem 2.10, we must show how the desired $C_{\alpha}$ 's are constructed. First, for a given $\alpha$, write $A_{\alpha+1}=A_{\alpha} \oplus D_{\alpha}$ where $D_{\alpha}$ is generated by an appropriate subset of $X$. Next choose $D_{\alpha}^{\prime}$ maximal in $D_{\alpha}$ with respect to satisfying the condition $\left(A_{\alpha} \cap H\right) \oplus\left(A_{\alpha} \cap K\right) \oplus D_{\alpha}^{\prime} \subseteq$ $\left(A_{\alpha+1} \cap H\right) \oplus\left(A_{\alpha+1} \cap K\right)$. Routine arguments show that both $D_{\alpha} / D_{\alpha}^{\prime}$ and

$$
\left(\left(A_{\alpha+1} \cap H\right) \oplus\left(A_{\alpha+1} \cap K\right)\right) /\left(\left(A_{\alpha} \cap H\right) \oplus\left(A_{\alpha} \cap K\right) \oplus D_{\alpha}^{\prime}\right)
$$

are torsion groups. Now set $C_{\alpha}=\left(A_{\alpha+1} \cap H\right) \cap\left[\left(A_{\alpha} \cap K\right) \oplus D_{\alpha}^{\prime}\right]$. Clearly $\left(A_{\alpha} \cap H\right) \oplus C_{\alpha}$ is a *-valuated coproduct and it is a simple matter to see that $\left(A_{\alpha+1} \cap H\right) /\left(\left(A_{\alpha} \cap H\right) \oplus C_{\alpha}\right)$ is also torsion. Finally, it is evident that $C_{\alpha}$ is countable since $A_{\alpha+1} / A_{\alpha}$ is, and the fact that $C_{\alpha}$ is $k$-basic in $G$ is a consequence of Proposition 2.2 and two applications of Remark 2.9.

\section{AXIOM 3 AND WARFIELD GROUPS}

A mixed group $G$ is said to satisfy Axiom 3 with respect to knice subgroups if there exists a collection $\mathscr{C}$ of knice subgroups of $G$ possessing the following properties:

(0) $0 \in \mathscr{C}$.

(1) $\mathscr{C}$ is closed with respect to arbitrary group unions.

(2) If $C \in \mathscr{C}$ and if $S$ is an arbitrary countable subset of $G$, then there is a countable subgroup $A$ containing $S$ such that $C+A$ belongs to $\mathscr{C}$.

We shall say that $G$ satisfies Griffith's version of Axiom 3 [G] with respect to knice subgroups provided there exists a collection $\mathscr{C}$ of knice subgroups of $G$ with properties (0) and (2) above and also the following weakened version of condition (1):

(1') $\mathscr{C}$ is closed with respect to unions of chains.

That summands of $k$-groups are also $k$-groups, Theorem 2.6 , is crucial to our next result, and consequently this fact about $k$-groups plays an indispensable role in our Axiom 3 characterization of global Warfield groups.

Theorem 3.1. If the mixed group $G$ satisfies Axiom 3 with respect to knice subgroups, then so does any direct summand.

Proof. Suppose that $G=H \oplus K$ and let $\mathscr{C}$ be an Axiom 3 family of knice subgroups of $G$. We then take $\mathscr{C}^{\prime}$ to consist of all subgroups $A$ of $H$ for which there corresponds a $B \in \mathscr{C}$ such that $B=A \oplus(B \cap K)$. It is well documented (see Lemma 81.5 in [F3]) that $\mathscr{C}^{\prime}$ satisfies conditions (0), (1), (2) above, and therefore it remains only to show that the members of $\mathscr{C}^{\prime}$ are knice subgroups of $H$. That each $A \in \mathscr{C}^{\prime}$ is a nice subgroup of $G$ follows from Proposition 1.11 in [HM4] and niceness in $H$ is then evident since $H$ is an isotype subgroup 
of $G$. Suppose $A \in \mathscr{C}^{\prime}$ and $B=A \oplus(B \cap K)$ is in $\mathscr{C}$. Then obviously $G / B \cong H / A \oplus K /(B \cap K)$ and, by Theorem $2.6, H / A$ is a $k$-group since $G / B$ is. Notice furthermore that if $x \in H$, then $\|x+B\|_{G / B}=\|x+A\|_{G / A}=\|x+A\|_{H / A}$. Consequently an application of Proposition 1.7 establishes the fact that $A$ is a knice subgroup.

We are now in position to prove the main theorem of this paper, an omnibus result establishing a variety of characterizations of global Warfield groups. Each item in the next theorem, of course, generalizes some well-known characterization of totally projective groups.

Theorem 3.2. For an arbitrary mixed group $G$, the following conditions are equivalent:

(i) $G$ satisfies Axiom 3 with respect to knice subgroups.

(ii) $G$ satisfies Griffith's version of Axiom 3 with respect to knice subgroups.

(iii) $G$ is the union of a smooth well-ordered chain $\left(G_{\alpha}\right)_{\alpha<\tau}$ of nice subgroups such that $G_{0}=0$ and, for each $\alpha$, either $G_{\alpha+1} / G_{\alpha}$ is cyclic of prime order or else $G_{\alpha+1}=G_{\alpha} \oplus\left\langle x_{\alpha}\right\rangle$ is a valuated coproduct in $G$ with $x_{\alpha}$ an element of infinite order.

(iv) $G$ is sequentially pure projective.

(v) $G$ is a direct summand of a simply presented group.

(vi) $G$ has a decomposition basis and satisfies Axiom 3 with respect to nice subgroups.

Proof. It is, of course, trivial that (i) implies (ii). Now assume that (ii) is satisfied with $\mathscr{C}$ a family of knice subgroups enjoying properties $(0),\left(1^{\prime}\right),(2)$. Let $\left(g_{\beta}\right)_{\beta<\mu}$ be a well ordering of the elements of $G$. It is obvious that there is a smooth chain of subgroups $A_{\beta} \in \mathscr{C}, g_{\beta} \in A_{\beta+1}$, and $A_{\beta+1} / A_{\beta}$ is countable. We, however, need to place further restrictions on the $A_{\beta}$ 's, namely, we wish to associate with each $A_{\beta}$ a countable subset $Y_{\beta}$ of primitive elements such that $A_{\beta}^{\prime}=A_{\beta} \oplus\left(\bigoplus_{y \in Y_{\beta}}\langle y\rangle\right)$ is a *-valuated coproduct with $A_{\beta+1} / A_{\beta}^{\prime}$ torsion. The achievement of this further restriction on the transition from $A_{\beta}$ to $A_{\beta+1}$ involves a routine back-and-forth argument in which one repeatedly utilizes condition (2) for $\mathscr{C}$ and Proposition 1.6(2), with $A_{\beta+1}$ obtained via condition $\left(1^{\prime}\right)$ by taking the union of an ascending sequence of members of $\mathscr{C}$ that arise in the aforementioned back-and-forth construction. The final step, in showing that (iii) is a consequence of (ii), is to refine the $A_{\beta}$ 's to obtain the $G_{\alpha}$ 's in such a fashion that, when $\alpha$ is a limit ordinal, $G_{\alpha}$ equals some $A_{\beta}$. In the intercalation between $G_{\alpha}=A_{\beta}$ and $G_{\alpha+\omega}=A_{\beta+1}$, we need to avail ourselves of Proposition 1.6(1) and the Seesaw Lemma, Proposition 2.7 of [HM4].

Next we prove that (iii) implies (iv). Assume then that $0 \rightarrow A \stackrel{\mu}{\rightarrow} B \stackrel{\sigma}{\rightarrow} C \rightarrow 0$ is a sequentially pure short exact sequence and that $\theta: G \rightarrow C$ is an epimorphism. We shall use the $G_{\alpha}$ 's to construct inductively a homomorphism $\pi: G \rightarrow B$ such that $\sigma \pi=\theta$. Indeed suppose we have already constructed a homomorphism $\pi_{\alpha}: G_{\alpha} \rightarrow B$ such that, for all $g \in G_{\alpha}, \sigma \pi_{\alpha}(g)=\theta(g)$, and $\|g\|_{G} \leq\left\|\pi_{\alpha}(g)\right\|_{B}$. First consider the case where $G_{\alpha+1} / G_{\alpha}$ is cyclic of order $p$. Since $G_{\alpha}$ is a nice subgroup of $G$, there is an element $x_{\alpha} \in G_{\alpha+1}$ such that $G_{\alpha+1}=\left\langle x_{\alpha}, G_{\alpha}\right\rangle$ and $\left|x_{\alpha}\right|_{p} \geq\left|x_{\alpha}+g\right|_{p}$ for all $g \in G_{\alpha}$. Then select $b \in B$ so that $\sigma(b)=\theta\left(x_{\alpha}\right)$ and $\|b\|_{B}=\left\|\theta\left(x_{\alpha}\right)\right\|_{C} \geq\left\|x_{\alpha}\right\|_{G}=M$. Because $p b-\pi_{\alpha}\left(p x_{\alpha}\right) \in \operatorname{Ker} \sigma \cap B(p M)=\mu(A(p M))$, there is an $a \in A(M)$ 
such that $p(b-\mu(a))=\pi_{\alpha}\left(p x_{\alpha}\right)$. Take $y=b-\mu(a)$ and observe that $\sigma(y)=\theta\left(x_{\alpha}\right),\|y\|_{B} \geq\left\|x_{\alpha}\right\|_{G}$, and $\pi_{\alpha}\left(p x_{\alpha}\right)=p y$. Since the elements $z$ of $G_{\alpha+1}$ are uniquely representable in the form $z=k x_{\alpha}+g$ where $0 \leq k<p$ and $g \in G_{\alpha}$, we obtain a homomorphism $\pi_{\alpha+1}: G_{\alpha+1} \rightarrow B$ extending $\pi_{\alpha}$ by defining $\pi_{\alpha+1}(z)=k y+\pi_{\alpha}(g)$. Clearly then $\sigma \pi_{\alpha+1}(z)=\theta(z)$ for all $z \in C_{\alpha+1}$. Moreover $\|z\|_{G} \leq\left\|\pi_{\alpha+1}(z)\right\|_{B}$ because $\|p z\|_{G} \leq\left\|\pi_{\alpha}(p z)\right\|_{B}=\left\|\pi_{\alpha+1}(p z)\right\|_{B}$ and $\left|\pi_{\alpha+1}(z)\right|_{p} \geq|k y|_{p} \wedge\left|\pi_{\alpha}(g)\right|_{p} \geq\left|k x_{\alpha}\right|_{p} \wedge|g|_{p}=\left|k x_{\alpha}+g\right|_{p}=|z|_{p}$. It remains to consider the case where $G_{\alpha+1}=G_{\alpha} \oplus\left\langle x_{\alpha}\right\rangle$ is a valuated coproduct. In this instance we need only choose $y \in B$ so that $\sigma(y)=\theta\left(x_{\alpha}\right)$ and $\|y\|_{B}=\left\|\theta\left(x_{\alpha}\right)\right\|_{C} \geq\left\|x_{\alpha}\right\|_{G}$ and define $\pi_{\alpha+1}: G_{\alpha+1} \rightarrow B$ by taking

$$
\pi_{\alpha+1}\left(k x_{\alpha}+g\right)=k y+\pi_{\alpha}(g)
$$

whenever $k$ is an integer and $g \in G_{\alpha}$. Obviously $\pi_{\alpha+1}$ is a homomorphism extending $\pi_{\alpha}$. Moreover, for all $z=k x_{\alpha}+g$ in $G_{\alpha+1}, \sigma \pi_{\alpha+1}(z)=\theta(z)$ and $\left\|\pi_{\alpha+1}(z)\right\|_{B}=\left\|k y+\pi_{\alpha}(g)\right\|_{B} \geq\|k y\|_{B} \wedge\left\|\pi_{\alpha}(g)\right\|_{B} \geq\left\|k x_{\alpha}\right\|_{G} \wedge\|g\|_{G}=$ $\left\|k x_{\alpha}+g\right\|_{G}=\|z\|_{G}$.

The fact that (iv) implies (v) is due to Warfield [W] and we shall merely sketch his proof. Take $B$ to be the abelian group generated by $X=G$ subject only to those relations of the form $n x=y$ and $n x=0$ that happen to hold in $G$. Then $B$ is simply presented and there is an epimorphism $\sigma: B \rightarrow G$ induced by the correspondence $x \rightarrow x$. One checks that the associated short exact sequence $0 \rightarrow \operatorname{Ker} \sigma \rightarrow B \stackrel{\sigma}{\rightarrow} G \rightarrow 0$ is sequentially pure and then concludes, by the hypothesis, that $G$ is isomorphic to a direct summand of $B$.

We now prove that (v) implies (i). By Theorem 3.1, it suffices to show that each simply presented mixed abelian group $G$ satisfies Axiom 3 for knice subgroups. Recall that if $G$ is a simply presented mixed group, then $G=$ $\bigoplus_{i \in I} G_{i}$ where each $G_{i}$ is rank 1 and simply presented [W]. Moreover, by a standard argument (see Lemma 81.5 of [F3]) it is readily seen that $G=\bigoplus_{i \in I} G_{i}$ will satisfy Axiom 3 for knice subgroups provided each $G_{i}$ does. Thus we have reduced the proof to the situation where $G$ is a rank 1 simply presented group, in which case there is an element $x$ of infinite order such that $G /\langle x\rangle$ is a simply presented torsion group [W]; that is, $G /\langle x\rangle$ satisfies Axiom 3 for nice subgroups. Clearly then $G$ is a $k$-group and $x$ is a primitive element. If $\mathscr{C}$ consists of 0 and all the subgroups $A$ such that the quotients $A /\langle x\rangle$ form an Axiom 3 collection of nice subgroups of $G /\langle x\rangle$, then the members of $\mathscr{C}$ are knice by Proposition 1.6(4) and consequently $G$ satisfies Axiom 3 for knice subgroups.

We have thus far skipped (vi) in our cycle of implications. But notice that the $x_{\alpha}$ 's that occur in the statement of (iii) obviously form a decomposition basis for $G=\bigcup_{\alpha<\mu} G_{\alpha}$. Since (i) implies (iii), we know that condition (vi) holds for any mixed group $G$ that satisfies Axiom 3 with respect to knice subgroups. Finally, we show that (vi) implies (ii). Assume that $X$ is a decomposition basis for the mixed group $G$ and let $\mathscr{C}$ be a family of nice subgroups of $G$ that satisfies conditions (0), (1), and (2) above. We say that subgroups $A$ and $B$ of $G$ are locally compatible (and we write $A \| B$ ) provided, for each prime $p$ and each ordinal $\alpha,(A+B) \cap p^{\alpha} G=\left(A \cap p^{\alpha} G\right)+\left(B \cap p^{\alpha} G\right)$. Now take $\mathscr{C}^{\prime}$ to consist of all subgroups $A$ of $G$ that satisfy the following three conditions:

(a) $A$ is a pure subgroup of $G$ with $A \in \mathscr{C}$.

(b) $A \| F$ where $F=\langle X\rangle$. 
(c) $A /\left\langle X_{A}\right\rangle$ is torsion where $X_{A}=X \cap A$.

We claim that $\mathscr{C}^{\prime}$ is a family of knice subgroups of $G$ that satisfies conditions $(0),\left(1^{\prime}\right)$, and (2). It is clear, of course, that $\mathscr{C}^{\prime}$ satisfies $(0)$ and $\left(1^{\prime}\right)$. To see that the members of $\mathscr{C}^{\prime}$ are knice subgroups of $G$ it suffices to show that, for each $A \in \mathscr{C}^{\prime}, A \oplus\left\langle Y_{A}\right\rangle$ is a *-valuated coproduct where $Y_{A}=X \backslash X_{A}$. In fact, since $A /\left\langle X_{A}\right\rangle$ is torsion and $F=\left\langle X_{A}\right\rangle \oplus\left\langle Y_{A}\right\rangle$ is a *-valuated coproduct, we need only prove that $A \oplus\left\langle Y_{A}\right\rangle$ is a valuated coproduct. But this is an easy consequence of condition (b) and the fact that $F=\bigoplus_{x \in X}\langle x\rangle$ is a valuated coproduct. The proof that the family $\mathscr{C}^{\prime}$ satisfies condition (2) requires some further notions. Recall that a subgroup $H$ of $G$ is said to be separable (in the sense of Hill) if for each $g \in G$ there is a countable subset $\left(h_{n}\right)_{n<\omega}$ of $H$ satisfying the following condition: for $h \in H$ there is a corresponding $n$ such that $|g+h| \leq\left|g+h_{n}\right|$. The proof of Theorem 3.1 in [HM6] is easily adapted to establish the following fact: If $F=\langle X\rangle$ where $X$ is a decomposition basis for $G$, then $F$ is separable in $G$. Since $F$ is separable in $G$, each countable subset $S$ of $G$ is contained in some countable subgroup $A$ with $A \| F$. Indeed $A$ can be chosen to satisfy the following stronger condition: If $s$ is an abstract height, then $(A+F) \cap G(s)=(A \cap G(s))+(F \cap G(s))$ where $G(s)=\{x \in G:|x| \geq s\}$. (See the proof of Lemma 5.3 in [HM6].) To pass beyond the countability limitations of the construction of an $A$ with $A \| F$, we need the following easily proved observation: If $A$ is a pure and knice subgroup of $G$ with $A \| F$ and if $B / A \|(F+A) / A$, then $B \| F$. (Notice that Corollary 1.10 is crucial to the proof.) In order to exploit this last observation, one needs to note that when $A$ satisfies conditions (a), (b), and (c), then $(F+A) / A$ is separable in $G / A$ because $(x+A)_{x \in Y_{A}}$ is a decomposition basis by Remark 1.8. Combining these observations concerning separability and local compatibility with the fact that every countable extension of a pure subgroup $A$ is contained in a pure subgroup $B$ with $B / A$ countable, one can show by a straightforward back-andforth argument that $\mathscr{C}^{\prime}$ does indeed satisfy condition (2). This completes the proof of Theorem 3.2.

Corollary 3.3. Let $G$ be a mixed group of cardinality $\aleph_{1}$. Then $G$ is a Warfield group if and only if there is a collection $\mathscr{C}$ of knice subgroups of $G$ that satisfies the following three conditions:

(0) $0 \in \mathscr{C}$.

(1") $\mathscr{C}$ is closed under the union of countable ascending chains. $\mathscr{C}$.

$\left(2^{\prime}\right)$ Each countable subset of $G$ is contained in some countable member of

Proof. Since $|G|=\aleph_{1}$, it is routine to extract a well-ordered chain $\mathscr{C}^{\prime} \subseteq \mathscr{C}$ such that $\mathscr{C}^{\prime} \cup\{G\}$ is a family of knice subgroups of $G$ satisfying the requisite conditions (0), (1), and (2) of an Axiom 3 family.

Corollary 3.4. If $H$ is an isotype knice subgroup of the mixed group $G$ with $G / H$ countable, then $H$ is a direct summand of $G$.

Proof. By Proposition 1.9, $H$ is sequentially pure in $G$ and $G / H$ is a $k$-group. Since Theorem 3.2, howver, implies that countable $k$-groups are sequentially pure projective, the desired conclusion follows. 


\section{SUbGRoups OF WARFIELD GROUPS}

The Axiom 3 characterization of Warfield groups provides a tool for the study of isotype subgroups of simply presented groups having the potential to yield results not accessible by any of the other known characterizations of these groups. Illustrations, in more specialized contexts, of such results may be found in [HM1, HM2, HM6, HM7, H2 and HLM]. Since we plan to deal further with such applications in subsequent publications, we limit ourselves here to the following result.

Theorem 4.1. If $H$ is an isotype knice subgroup of the Warfield group $G$ with $|G / H| \leq \aleph_{1}$, then $H$ itself is a Warfield group.

In view of Proposition 1.9, this theorem says that a $k$-group of cardinality $\aleph_{1}$ has sequentially pure projective dimension $\leq 1$. The corresponding theorems for $p$-groups and torsion-free groups appear in [FH and DR], respectively. We shall require a couple of preliminary lemmas before we can prove the theorem above.

Lemma 4.2. Let $H$ be an isotype knice subgroup of the mixed group $G$. If $A$ is a knice subgroup of $G$ with $A \subseteq H$, then $A$ is a knice subgroup of $H$.

Proof. First we show that $H / A$ is a knice subgroup of $G / A$. Actually $H / A$ satisfies the strong version of niceness in $G / A$, that is, $p^{\alpha}((G / A) /(H / A))=$ $\left(p^{\alpha}(G / A)+(H / A)\right) /(H / A)$ for all primes $p$ and all ordinals $\alpha$. Indeed this follows from Corollary 1.10 and the proof of Lemma 79.3 in [F3]. That part (2) of Definition 1.5 holds for $H / A$ follows from Remark 1.8. Next we show that $H / A$ is isotype in $G / A$. Suppose, for some prime $p$ and some ordinal $\alpha$, that $x+A \in p^{\alpha}(G / A)$ where $x \in H$. By Proposition 1.7, there is a positive integer $n$ and an element $a$ in $A$ such that $\|n x+a\|_{G}=\|n x+A\|_{G / A}$. Let $n=p^{i} m$ where $(m, p)=1$. Then, since $A$ is a nice subgroup of $G, m x$ is in $p^{\alpha} G+A$ and therefore there is an $a^{\prime} \in A$ such that $h=m x+a^{\prime} \in p^{\alpha} G \cap H=p^{\alpha} H$. Thus $m x+A$ is in $p^{\alpha}(H / A)$ and hence $x+A \in p^{\alpha}(H / A)$ because $(m, p)=1$. It now follows from Theorem 2.8 that $H / A$ is a $k$-group. It remains to show that $A$ satisfies, relative to $H$, conditions (2) and (3) of Proposition 1.7. That $A$ is a nice subgroup of $H$ follows easily from the fact that $A$ is nice in $G$ and $H$ is an isotype subgroup. Condition (3) of Proposition 1.7 holds for $A$ and $H$ because $H / A$ is isotype in $G / A$ and this condition is satisfied for $A$ and $G$.

We say that the subgroups $A$ and $H$ of the mixed group $G$ are strongly compatible provided, for each height matrix $M,(A+H) \cap G(M)=(A \cap G(M))+$ $(H \cap G(M))$. An equivalent formulation is that for each pair $(a, h) \in A \times H$ there exists an element $a^{\prime} \in A \cap H$ such that $\left\|a^{\prime}+h\right\| \geq\|a+h\|$.

Lemma 4.3. Let $H$ be an isotype knice subgroup of the mixed group $G$. If $A$ is a knice subgroup of $G$ such that each finite subset of $(A+H) / H$ is torsion modulo a subgroup that is $k$-basic in $G / H$ and the subgroups $A$ and $H$ are strongly compatible, then $A \cap H$ is a knice subgroup of $G$.

Proof. We shall demonstrate that $A \cap H$ satisfies the three requisite conditions of Proposition 1.7. In doing so, we will need to exploit the fact that $A$ and $H$ are strongly compatible in three separate contexts. But in each instance we will have elements $a \in A$ and $h \in H$, and we will choose $a_{1} \in A$ and 
$h_{1} \in H$ such that $a_{1}-h_{1}=a-h$ and $\left\|a_{1}\right\| \wedge\left\|h_{1}\right\| \geq\|a-h\|$. To show that $A \cap H$ is a nice subgroup of $G$, we assume that $x+(A \cap H) \in p^{\alpha}(G / A \cap H)$ and $p x \in p^{\alpha} G+(A \cap H)$ where $p$ is an arbitrary prime and $\alpha$ an ordinal. Since $H$ and $A$ are both nice in $G$, we can write $x=z_{1}+h=z_{2}+a$ where $z_{1}, z_{2} \in p^{\alpha} G, a \in A$, and $h \in H$. Then with $a_{1}$ and $h_{1}$ chosen as above, we have $h_{1} \in p^{\alpha} G$ and therefore $x=\left(z_{1}+h_{1}\right)+\left(a-a_{1}\right)$ where $z_{1}+h_{1}$ is in $p^{\alpha} G$ and $a-a_{1}=h-h_{1} \in A \cap H$. Thus, $A \cap H$ is also nice in $G$. Next we show that condition (3) of Proposition 1.7 holds for $A \cap H$. Given $g \in G$ there is, since $A$ is a knice subgroup of $G$, a positive integer $n$ such that $x=n g$ satisfies $\|x+A\|=\|x+a\|$ for some appropriate $a \in A$. But then, because $H$ is sequentially pure in $G$, there is an $h \in H$ with $\|x+h\|=\|x+H\|$. Again choosing $a_{1}$ and $h_{1}$ as above, we maintain that $a-a_{1}=h-h_{1}$ is an element of $A \cap H$ such that

$$
\left\|x+\left(a-a_{1}\right)\right\|=\|x+(A \cap H)\|=\|x+A\|=\|x+H\| .
$$

Indeed this follows from the observation that $\left\|a_{1}\right\| \wedge\left\|h_{1}\right\| \geq\|a-h\| \geq\|x+a\| \wedge$ $\|x+h\|$ and therefore

$$
\begin{aligned}
\left\|x+\left(a-a_{1}\right)\right\| & \geq\left(\|x+a\| \wedge\left\|a_{1}\right\|\right) \wedge\left(\|x+h\| \wedge\left\|h_{1}\right\|\right) \\
& =\|x+A\| \wedge\|x+H\| \geq\|x+(A \cap H)\| .
\end{aligned}
$$

That $G /(A \cap H)$ is a $k$-group depends on the structure of $(A+H) / H$ as a subgroup of $G / H$ and the crucial fact that the canonical isomorphism between $(A+H) / H$ and $A /(A \cap H)$ preserves height matrices as computed in $G / H$ and $G /(A \cap H)$, respectively; that is, $\|a+H\|=\|a+(A \cap H)\|$ for all $a \in A$. To substantiate this latter claim, we utilize the fact that $H$ is sequentially pure in $G$ to choose an $h \in H$ such that $\|a-h\|=\|a+H\|$. Once more select $a_{1}$ and $h_{1}$ as in the preceding paragraph. Then $\left(h_{1}-h\right) \in A \cap H$ and

$\|a+H\|=\|a-h\|=\|a-h\| \wedge\left\|h_{1}\right\| \leq\left\|a+\left(h_{1}-h\right)\right\| \leq\|a+(A \cap H)\| \leq\|a+H\|$.

As a consequence of this preservation of height matrices, a subgroup $k$-basic in $G / H$ and contained in $(A+H) / H$ will map canonically to a subgroup $k$-basic in $G /(A \cap H)$, and hence $A /(A \cap H)$ inherits from $(A+H) / H$ the property of finite subsets being torsion modulo a subgroup that is $k$-basic. Now to complete the proof that $G /(A \cap H)$ is a $k$-group, we select an arbitrary finite subset $\bar{S}$ of $G /(A \cap H)$ and then pick a finite subset $S$ of $G$ mapping onto it under the natural projection from $G$ onto $G /(A \cap H)$. Since $A$ is a knice subgroup of $G$, there are primitive elements $x_{1}, \ldots, x_{m}$ in $G$ such that $S$ is finite modulo the *-valuated coproduct $A \oplus\left\langle x_{1}\right\rangle \oplus \cdots \oplus\left\langle x_{m}\right\rangle$. But then, by Remark 1.8, the $x_{i}+(A \cap H)$ 's are primitive in $G /(A \cap H)$ and $(A /(A \cap H)) \oplus\left\langle x_{1}+(A \cap H)\right\rangle \oplus \cdots \oplus\left\langle x_{m}+(A \cap H)\right\rangle$ is a *-valuated coproduct. Finally, taking advantage of the structure of $A /(A \cap H)$, we can select primitive elements $y_{1}+(A \cap H), \ldots, y_{n}+(A \cap H)$ of $G /(A \cap H)$ where the $y_{j}$ 's are in $A$ and $\bar{S}$ is finite modulo the *-valuated coproduct

$$
\left\langle y_{1}+(A \cap H)\right\rangle \oplus \cdots \oplus\left\langle y_{n}+(A \cap H)\right\rangle \oplus\left\langle x_{1}+(A \cap H)\right\rangle \oplus \cdots \oplus\left\langle x_{m}+(A \cap H)\right\rangle .
$$

We are now in position to prove Theorem 4.1. First note that we may assume without loss of generality that $|G|=\aleph_{1}$; the point here is the Schanuel's trick is available since sequential purity is preserved under the pullback construction and direct summands of Warfield groups are, of course, Warfield groups. Next 
we observe, since $G / K$ is a $k$-group of cardinality of most $\aleph_{1}$, that $G / K$ is the union of a family $\mathscr{D}$ of countable subgroups satisfying the following two conditions:

(1) Each member of $\mathscr{D}$ has the property that its finite subsets are torsion modulo a subgroup that is $k$-basic in $G / H$.

(2) $0 \in \mathscr{D}$ and $\mathscr{D}$ is closed under the union of countable ascending chains. Indeed, taking advantage of Proposition 1.6(2), the construction of $\mathscr{D}$ is substantially that given in the first paragraph of the proof of Theorem 5.4 in [HM3]. Then, by Corollary 3.3, we also see that $G$ is the union of a family $\mathscr{C}$ of countable subgroups satisfying the following two conditions:

(3) Each member of $\mathscr{C}$ is a knice subgroup of $G$.

(4) $0 \in \mathscr{D}$ and $\mathscr{C}$ is closed under the union of countable ascending chains.

Utilizing the fact that $H$ is sequentially pure in $G$, one easily adapts the proof of Lemma 5.3 in [HM6] to prove the following:

(5) Each countable subset of $G$ is embeddable in a countable subgroup $A$ with $A$ and $H$ strongly compatible.

With these preliminaries disposed cf, we introduce the family $\mathscr{C}^{\prime}$ consisting of those subgroups $A$ of $G$ that satisfy (i) $A$ and $H$ are strongly compatible, (ii) $A \in \mathscr{C}$ and (iii) $(A+H) / H$ is in $\mathscr{D}$. A routine interlacing, back-and-forth argument exploiting (2), (4), and (5) shows that $\mathscr{C}^{\prime}$ is closed under the union of countable ascending chains and that $G$ is the union of the subgroups in $\mathscr{C}^{\prime}$. Therefore the family $\mathscr{C}^{\prime} \cap H=\left\{A \cap H: A \in \mathscr{C}^{\prime}\right\}$ enjoys the same properties relative to $H$. Once we establish the fact that each subgroup in $\mathscr{C}^{\prime} \cap H$ is knice in $H$, then Corollary 3.3 will imply that $H$ is Warfield group. But if $A \in \mathscr{C}^{\prime}$, then $A \cap H$ is knice in $G$ by Lemma 4.3 and thus knice in $H$ by Lemma 4.2.

\section{REFERENCES}

[AHR] D. Arnold, R. Hunter, and F. Richman, Global Azumaya theorems in additive categories, J. Pure Appl. Algebra 16 (1980), 223-243.

[B] R. Baer, Abelian groups without elements of infinite order, Duke Math. J. 3 (1937), 68-122.

[DR] M. Dugas and K. Rangaswamy, On torsion-free abelian k-groups, Proc. Amer. Math. Soc. 99 (1987), 403-408.

[F1] L. Fuchs, Abelian groups, Publishing House of the Hungarian Academy of Sciences, Budapest, 1958.

[F2] _ Summands of separable abelian groups, Bull. London Math. Soc. 2 (1970), 205-208.

[F3] _ Infinite Abelian groups, vol. II, Academic Press, New York, 1973.

[FH] L. Fuchs and P. Hill, The balanced-projective dimension of abelian p-groups, Trans. Amer. Math. Soc. 293 (1986), 99-112.

[G] P. Griffith, Infinite Abelian groups, Univ. of Chicago Press, Chicago, Ill., 1970.

[H1] P. Hill, On the classification of abelian groups, Photocopied manuscript, 1967.

[H2] _ Isotype subgroups of totally projective groups, Lecture Notes in Math., vol. 874, Springer-Verlag, New York, 1981, pp. 305-321.

[HLM] P. Hill, M. Lane, and C. Megibben, On the structure of p-local groups, J. Algebra 143 (1991), 29-45.

[HM1] P. Hill and C. Megibben, On the theory and classification of abelian p-groups, Math. Z. 190 (1985), 17-38.

[HM2] _ Axiom 3 modules, Trans. Amer. Math. Soc. 295 (1986), 715-734. 
[HM3] _ , Torsion free groups, Trans. Amer. Math. Soc. 295 (1986), 735-751.

[HM4] _ Knice subgroups of mixed groups, Abelian Group Theory, Gordon-Breach, New York, 1987, pp. 89-109.

[HM5] _ Ten lectures on abelian groups, Workshop on Abelian Groups, Univ. of Colorado at Colorado Springs, 1986.

[HM6] _-, Pure subgroups of torsion free groups, Trans. Amer. Math. Soc. 303 (1987), 765-778.

[HM7] _ The local equivalence theorem, Contemp. Math. 87 (1989), 201-219.

[HM8] _ Generalizations of the stacked bases theorem, Trans. Amer. Math. Soc. 312 (1989), 377-402.

[HM9] — The classification of certain Butler group, J. Algebra (to appear).

[HR] R. Hunter and F. Richman, Global Warfield groups, Trans. Amer. Math. Soc. 266 (1981), 555-572.

[HRW1] R. Hunter, F. Richman, and E. Walker, Warfield modules, Lecture Notes in Math., vol. 616, Springer-Verlag, New York, 1977, pp. 87-123.

[HRW2] __, Existence theorems for Warfield groups, Trans. Amer. Math. Soc. 235 (1978), 345362.

[S] R. Stanton, Almost-affable abelian groups, J. Pure Appl. Algebra 15 (1979), 41-52.

[W] R. Warfield, Simply presented groups, Proc. Sem. Abelian Group Theory, Univ. of Arizona lecture notes, 1972.

Department of Mathematics, Auburn University, Auburn, Alabama 36849

Department of Mathematics, Vanderbilt University, NashVille, Tennessee 37235 